\title{
Degenerate elliptic equations with nonlinear boundary conditions and measures data
}

\author{
Fuensanta Andreu, Noureddine Igbida, José M. Mazón \\ AND JULIÁN TOLEDO
}

Dedicated to our friend Lucio Boccardo on the occasion of his 60th birthday.

\begin{abstract}
In this paper we study the questions of existence and uniqueness of solutions for equations of type $-\operatorname{div} \mathbf{a}(x, D u)+\gamma(u) \ni \mu_{1}$, posed in an open bounded subset $\Omega$ of $\mathbb{R}^{N}$, with nonlinear boundary conditions of the form $\mathbf{a}(x, D u) \cdot \eta+\beta(u) \ni \mu_{2}$. The nonlinear elliptic operator $\operatorname{div} \mathbf{a}(x, D u)$ is modeled on the $p$-Laplacian operator $\Delta_{p}(u)=\operatorname{div}\left(|D u|^{p-2} D u\right)$, with $p>1, \gamma$ and $\beta$ are maximal monotone graphs in $\mathbb{R}^{2}$ such that $0 \in \gamma(0) \cap \beta(0)$ and the data $\mu_{1}$ and $\mu_{2}$ are measures.
\end{abstract}

Mathematics Subject Classification (2000): $35 \mathrm{~J} 60$ (primary); 35D05 (secondary).

\section{Introduction}

The purpose of this paper is to establish existence and uniqueness of solutions for a degenerate elliptic problem with nonlinear boundary condition of the form

$$
\left(S_{\mu_{1}, \mu_{2}}^{\gamma, \beta}\right) \quad \begin{cases}-\operatorname{div} \mathbf{a}(x, D u)+\gamma(u) \ni \mu_{1} & \text { in } \Omega \\ \mathbf{a}(x, D u) \cdot \eta+\beta(u) \ni \mu_{2} & \text { on } \partial \Omega,\end{cases}
$$

where $\Omega$ is a bounded domain in $\mathbb{R}^{N}$ with smooth boundary $\partial \Omega$, the function a $: \Omega \times \mathbb{R}^{N} \rightarrow \mathbb{R}^{N}$ is a Carathéodory function with growth of order $p-1$ $(p>1)$ with respect to the gradient, satisfying the classical Leray-Lions conditions, $\eta$ is the unit outward normal on $\partial \Omega$ and $\mu_{1}, \mu_{2}$ are measures such that $\mu_{1}=\mu_{1}\left\llcorner\Omega, \quad \mu_{2}=\mu_{2}\left\llcorner\partial \Omega\right.\right.$ and $\mu_{1}+\mu_{2}$ is a diffuse measure (it does not charge sets of zero $p$-capacity). The nonlinearities $\gamma$ and $\beta$ are maximal monotone 
graphs in $\mathbb{R}^{2}$ (see, e.g., [16]), $0 \in \gamma(0) \cap \beta(0)$, satisfying rather general assumptions. In particular, they may be multivalued and this allows to include the Dirichlet condition (taking $\beta$ to be the monotone graph $D$ defined by $D(0)=\mathbb{R}$ ) and the non homogeneous Neumann boundary condition (taking $\beta$ to be the monotone graph $N$ defined by $N(r)=0$ for all $r \in \mathbb{R}$ ) as well as many other nonlinear fluxes on the boundary that occur in some problems in Mechanics and Physics (see, e.g., [25] or [15]). Note also that, since $\gamma$ may be multivalued, problems of type $\left(S_{\mu_{1}, \mu_{2}}^{\gamma, \beta}\right)$ appear in various phenomena with changes of state like the multiphase Stefan problem (cf. [21]) and in the weak formulation of the mathematical model of the so called Hele-Shaw problem ( $c f$. [23] and [26]).

In the particular case $\mathbf{a}(x, \xi)=\xi$, the problem $\left(S_{\mu_{1}, \mu_{2}}^{\gamma, \beta}\right)$ reads

$$
\left(L_{\mu_{1}, \mu_{2}}^{\gamma, \beta}\right) \quad \begin{cases}-\Delta u+\gamma(u) \ni \mu_{1} & \text { in } \Omega \\ \partial_{\eta} u+\beta(u) \ni \mu_{2} & \text { on } \partial \Omega,\end{cases}
$$

where $\partial_{\eta} u$ simply denotes the outward normal derivative of $u$. For this kind of problems in the homogeneous case, $\mu_{2} \equiv 0$, the pioneering works are the paper by H. Brezis [15], in which problem $\left(L_{\mu_{1}, 0}^{\gamma, \beta}\right)$ is studied for $\gamma$ the identity, $\beta$ a maximal monotone graph and $\mu_{1} \in L^{2}(\Omega)$, and the paper by H. Brezis and W. Strauss [20], in which problem $\left(L_{\mu_{1}, 0}^{\gamma, \beta}\right)$ is studied for $\mu_{1} \in L^{1}(\Omega)$ and $\gamma, \beta$ continuous nondecreasing functions from $\mathbb{R}$ into $\mathbb{R}$ with $\gamma^{\prime} \geq \epsilon>0$. These works were extended by Ph. Bénilan, M. G. Crandall and P. Sacks [9] to the case of any $\gamma$ and $\beta$ maximal monotone graphs in $\mathbb{R}^{2}$ such that $0 \in \gamma(0) \cap \beta(0)$.

In [1,2] and [4], the results of [9] are extended by proving the existence and uniqueness of weak (or entropy/renormalized) solutions for the general nonhomogeneous problem $\left(S_{\phi, \psi}^{\gamma, \beta}\right)$, with $\phi \in L^{1}(\Omega)$ and $\psi \in L^{1}(\partial \Omega)$, which is quite different from the homogeneous case. The arguments of the proofs are very connected to the nature of the nonlinearities $\gamma$ and $\beta$. More precisely, the following cases are studied separately,

(A) $D(\gamma)=\mathbb{R}$ and, $D(\beta)=\mathbb{R}$ or $\operatorname{div} \mathbf{a}(x, D u)=\Delta_{p}(u)$,

(B) $\psi \equiv 0$ and, $D(\beta)=\mathbb{R}$ or $\operatorname{div} \mathbf{a}(x, D u)=\Delta_{p}(u)$,

(C) $\mathbb{R} \neq \overline{D(\gamma)} \subset D(\beta)$ (the obstacle problem).

For the case where the data $\phi$ and $\psi$ are Radon measures, the problem is again different. Our aim in this paper is to extend some of the above results to this situation. There is a large literature on elliptic problems with measure data, mainly for the homogeneous Dirichlet problem and $\gamma \equiv 0$, that is, for the problem

$$
\left(S_{\mu, 0}^{0, D}\right) \quad \begin{cases}-\operatorname{div} \mathbf{a}(x, D u)=\mu & \text { in } \Omega \\ u=0 & \text { on } \partial \Omega .\end{cases}
$$


In the linear case, existence and uniqueness of solutions of $\left(S_{\mu, 0}^{0, D}\right)$ was obtained by G. Stampacchia [32] by duality techniques. In the nonlinear case the first attempt to solve problem $\left(S_{\mu, 0}^{0, D}\right)$ was done by L. Boccardo and T. Gallouët, who proved in [11] and [12] the existence of weak solutions of $\left(S_{\mu, 0}^{0, D}\right)$ under the assumption $p>2-\frac{1}{N}$. On the question of uniqueness, even for the particular case $\mu \in L^{1}(\Omega)$, the definition of weak solution is not enough in order to get uniqueness. It was necessary to find some extra conditions on the distributional solutions of $\left(S_{\mu, 0}^{0, D}\right)$ in order to ensure both existence and uniqueness. This was done by Ph. Bénilan et al., for the case of measures in $L^{1}(\Omega)$, by introducing the concept of entropy solution in [6], and by P. L. Lions and F. Murat in an unpublished paper where the concept of renormalized solution was introduced. For diffuse measures, that is, for measures in $L^{1}(\Omega)+W^{-1, p^{\prime}}(\Omega)$, the problem was solved by L. Boccardo, T. Gallouët and L. Orsina in [13] in the framework of entropy solutions, and for general measures by G. Dal Maso et al. in [22] in the framework of renormalized soltuions.

The study of the homogeneous Dirichlet problem for the Laplacian and $\gamma \not \equiv 0$ was initiated by $\mathrm{Ph}$. Bénilan and $\mathrm{H}$. Brezis in 1975 (see [7]) for the particular case $\gamma(r)=g_{p}(r):=|r|^{p-1} r$. They proved the existence of weak solutions of problem

$$
\left(L_{\mu, 0}^{\gamma, D}\right) \begin{cases}-\Delta u+\gamma(u)=\mu & \text { in } \Omega \\ u=0 & \text { on } \partial \Omega,\end{cases}
$$

for any measure $\mu$ if $p<\frac{N}{N-2}(N \geq 2)$, and non existence if $p \geq \frac{N}{N-2}(N \geq 3)$ for $\mu=\delta_{a}$, with $a \in \Omega$. Problem $\left(L_{\mu, 0}^{\gamma, D}\right)$ was also studied by P. Baras and M. Pierre [5]. Recently it has been studied by H. Brezis, M. Marcus and A. C. Ponce in [18], where the general case of a continuous nondecreasing nonlinearity $\gamma(r), \gamma(0)=0$, is dealt with (see also [10,33] for the particular case $\gamma(r)=e^{r}-1$ ). The same problem has been studied by H. Brezis and A. C. Ponce [19] in the case $\operatorname{Dom}(\gamma) \neq \mathbb{R}$ closed. The case $\operatorname{Dom}(\gamma) \neq \mathbb{R}$ open has been studied by L. Dupaigne, A. C. Ponce and A. Porretta [24].

The study of nonlinear equations involving measures as boundary condition was initiated by A. Gmira and L. Veron [27]. They proved the existence of weak solutions of problem

$$
(G V) \begin{cases}-\Delta u+|u|^{q-1} u=0 & \text { in } \Omega \\ u=\mu & \text { on } \partial \Omega,\end{cases}
$$

for any Radon measure $\mu$ on $\partial \Omega$ in the subcritical case $1<q<\frac{N+1}{N-1}$. In the supercritical case, $q \geq \frac{N+1}{N-1}$, existence of solutions no longer holds; for instance, the problem has no solution if the measure $\mu$ is concentrated at a single point. M. Marcus and L. Veron in [30] characterized the Radon measures $\mu$ on $\partial \Omega$ for which problem $(G V)$ has solution in the supercritical case, these measures are those that 
are absolutely continuous respect to the Bessel capacity $C_{\frac{2}{q}}, q^{\prime}$ on $\partial \Omega$. In the last years an extensive study of removable singularities and boundary traces for this type of problems has been done by M. Marcus and L. Veron (see [31] and the references therein).

The study of reduced measures initiated in [18] by H. Brezis, M. Marcus and A. C. Ponce for problem $\left(L_{\mu, 0}^{\gamma, D}\right)$ has been developed in [19] by H. Brezis and A. C. Ponce for problems of the form

$$
\begin{cases}-\Delta u+\gamma(u)=0 & \text { in } \Omega \\ u=\mu & \text { on } \partial \Omega\end{cases}
$$

where $\gamma: \mathbb{R} \rightarrow \mathbb{R}$ is a nondecreasing continuous function with $\gamma(r)=0$ for all $r \leq 0$. In that paper the authors make the observation that in all the above problems the equation in $\Omega$ is nonlinear but the boundary conditions is the usual Dirichlet boundary condition. They also point out that it would be interesting to investigate problems with nonlinear boundary conditions of type

$$
\left(L_{0, \mu}^{g_{1}, \beta}\right) \quad\left\{\begin{array}{l}
-\Delta u+u=0 \quad \text { in } \Omega \\
\frac{\partial u}{\partial \eta}+\beta(u) \ni \mu \text { on } \partial \Omega,
\end{array}\right.
$$

where $\beta$ is a maximal monotone graph in $\mathbb{R}^{2}$. Observe that this problem is a particular case of our general problem.

Let us briefly summarize the contents of the paper. In Section 2 we fix the notation and give some preliminaries. Section 3 deals with the different concepts of solution we use. The next section is dedicated to establish the existence and uniqueness results. Finally, the last section is devoted to the particular case of Dirichlet boundary conditions.

ACKNOWLEDGEMENTS. This work has been performed during the visit of the second author to the Universitat de València and the visits of the first, third and fourth authors to the Université de Picardie Jules Verne. They thank these institutions for their support and hospitality. The authors have been partially supported by the Spanish MEC and FEDER, project MTM2008-03176.

\section{Preliminaries}

Throughout the paper, $\Omega \subset \mathbb{R}$ is a bounded domain with smooth boundary $\partial \Omega$, $p>1, \gamma$ and $\beta$ are maximal monotone graphs in $\mathbb{R}^{2}$ such that $0 \in \gamma(0) \cap \beta(0)$ and the Carathéodory function a : $\Omega \times \mathbb{R}^{N} \rightarrow \mathbb{R}^{N}$ satisfies 
( $\left.H_{1}\right)$ there exists $\Lambda>0$ such that $\mathbf{a}(x, \xi) \cdot \xi \geq \Lambda|\xi|^{p}$ for a.e. $x \in \Omega$ and for all $\xi \in \mathbb{R}^{N}$,

$\left(H_{2}\right)$ there exists $\sigma>0$ and $\varrho \in L^{p^{\prime}}(\Omega)$ such that $|\mathbf{a}(x, \xi)| \leq \sigma\left(\varrho(x)+|\xi|^{p-1}\right)$ for a.e. $x \in \Omega$ and for all $\xi \in \mathbb{R}^{N}$, where $p^{\prime}=\frac{p}{p-1}$,

$\left(H_{3}\right)\left(\mathbf{a}\left(x, \xi_{1}\right)-\mathbf{a}\left(x, \xi_{2}\right)\right) \cdot\left(\xi_{1}-\xi_{2}\right)>0$ for a.e. $x \in \Omega$ and for all $\xi_{1}, \xi_{2} \in$ $\mathbb{R}^{N}, \xi_{1} \neq \xi_{2}$.

The hypotheses $\left(H_{1}\right)-\left(H_{3}\right)$ are classical in the study of nonlinear operators in divergence form (cf., [29]). The model example of a function a satisfying these hypotheses is $\mathbf{a}(x, \xi)=|\xi|^{p-2} \xi$. The corresponding operator is the $p$-Laplacian operator $\Delta_{p}(u)=\operatorname{div}\left(|D u|^{p-2} D u\right)$.

For $1 \leq p<+\infty, L^{p}(\Omega)$ and $W^{1, p}(\Omega)$ denote respectively the standard Lebesgue space and Sobolev space, and $W_{0}^{1, p}(\Omega)$ is the closure of $\mathcal{D}(\Omega)$ in $W^{1, p}(\Omega)$. For $u \in W^{1, p}(\Omega)$, we denote by $u$ or $\tau(u)$ the trace of $u$ on $\partial \Omega$ in the usual sense and by $W^{\frac{1}{p^{\prime}}, p}(\partial \Omega)$ the set $\tau\left(W^{1, p}(\Omega)\right)$. Recall that $\operatorname{Ker}(\tau)=W_{0}^{1, p}(\Omega)$.

We denote by $\mathcal{L}^{N}$ the $N$-dimensional Lebesgue measure of $\mathbb{R}^{N}$ and by $\mathcal{H}^{N-1}$ the $(N-1)$-dimensional Hausdorff measure.

For an open bounded set $U$ of $\mathbb{R}^{N}$, we define the $p$-capacity relative to $U$, $C_{p}(., U)$, in the following classical way. For any compact subset $K$ of $U$,

$$
C_{p}(K, U)=\inf \left\{\int_{U}|D u|^{p} ; u \in \mathcal{C}_{c}^{\infty}(U), u \geq \chi_{K}\right\},
$$

where $\chi_{K}$ is the characteristic function of $K$; we will use the convention that inf $\emptyset=$ $+\infty$. The $p$-capacity of any open subset $O \subset U$ is defined by

$$
C_{p}(O, U)=\sup \left\{C_{p}(K) ; K \subset O \text { compact }\right\} .
$$

Finally, the $p$-capacity of any Borel set $A \subset U$ is defined by

$$
C_{p}(A, U)=\inf \left\{C_{p}(O) ; O \subset A \text { open }\right\} .
$$

A function $u$ defined on $U$ is said to be cap $_{p}$-quasi-continuous in $A \subset U$ if for every $\varepsilon>0$, there exists an open set $B_{\epsilon} \subseteq U$ with $C_{p}\left(B_{\epsilon}, U\right)<\varepsilon$ such that the restriction of $u$ to $A \backslash B_{\epsilon}$ is continuous. It is well known that every function in $W^{1, p}(U)$ has a cap $p$-quasi-continuous representative, whose values are defined cap $_{p}$-quasi everywhere in $U$, that is, up to a subset of $U$ of zero $p$-capacity. When we are dealing with the pointwise values of a function $u \in W^{1, p}(U)$, we always identify $u$ with its cap $p$-quasi-continuous representative.

We denote

$$
\operatorname{sign}_{0}(r):=\left\{\begin{array}{ll}
1 & \text { if } r>0, \\
0 & \text { if } r=0, \\
-1 & \text { if } r<0,
\end{array} \quad \operatorname{sign}_{0}^{+}(r):= \begin{cases}1 & \text { if } r>0, \\
0 & \text { if } r \leq 0 .\end{cases}\right.
$$


For $k>0$,

$$
T_{k}(r):=\max \{-k, \min \{r, k\}\}, \quad r \in \mathbb{R}
$$

and

$$
T_{k}^{+}(r):=\min \left\{r^{+}, k\right\}, \quad r \in \mathbb{R} .
$$

In [6], the authors introduce the set

$$
\mathcal{T}^{1, p}(\Omega)=\left\{u: \Omega \longrightarrow \mathbb{R} \text { measurable such that } T_{k}(u) \in W^{1, p}(\Omega) \quad \forall k>0\right\} .
$$

They also prove that for a given $u \in \mathcal{T}^{1, p}(\Omega)$, there exists a unique (up to a.e. equivalence) measurable function $v: \Omega \rightarrow \mathbb{R}^{N}$ such that

$$
D T_{k}(u)=v \chi_{\{|v|<k\}} \quad \forall k>0 .
$$

This function $v$ will be denoted by $D u$. It is clear that if $u \in W^{1, p}(\Omega)$, then $v \in L^{p}(\Omega)$ and $v=D u$ in the usual sense.

As in [1], $\mathcal{T}_{\text {tr }}^{1, p}(\Omega)$ denotes the set of functions $u$ in $\mathcal{T}^{1, p}(\Omega)$ satisfying the following conditions, there exists a sequence $u_{n}$ in $W^{1, p}(\Omega)$ such that

(a) $u_{n}$ converges to $u$ a.e. in $\Omega$,

(b) $D T_{k}\left(u_{n}\right)$ converges to $D T_{k}(u)$ in $L^{1}(\Omega)$ for all $k>0$,

(c) there exists a finite measurable function $v$ on $\partial \Omega$, such that $u_{n}$ converges to $v$ a.e. in $\partial \Omega$.

The function $v$ is the trace of $u$ in the generalized sense introduced in [1]. In the sequel, the trace of $u \in \mathcal{T}_{\text {tr }}^{1, p}(\Omega)$ on $\partial \Omega$ will be denoted by $\operatorname{tr}(u)$ or $u$. Let us recall that in the case $u \in W^{1, p}(\Omega), \operatorname{tr}(u)$ coincides with the trace of $u, \tau(u)$, in the usual sense, and

$$
\operatorname{Ker}(\operatorname{tr})=\mathcal{T}_{0}^{1, p}(\Omega),
$$

the space introduced in [6] to study $\left(S_{\phi, 0}^{\gamma, D}\right)$. Moreover, for every $u \in \mathcal{T}_{\text {tr }}^{1, p}(\Omega)$ and $k>0, \tau\left(T_{k}(u)\right)=T_{k}(\operatorname{tr}(u))$. If $\phi \in W^{1, p}(\Omega) \cap L^{\infty}(\Omega)$, then $u-\phi \in \mathcal{T}_{\operatorname{tr}}^{1, p}(\Omega)$ and $\operatorname{tr}(u-\phi)=\operatorname{tr}(u)-\tau(\phi)$.

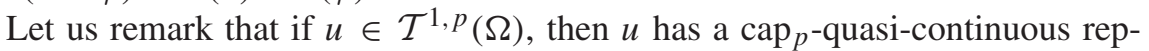
resentative, which will be denoted by $u$; the cap $p_{p}$-quasi-continuous representative can be infinite on a set of positive $p$-capacity (see [22]). If in addition the function $u \in \mathcal{T}^{1, p}(\Omega)$ is assumed to satisfy the estimate

$$
\int_{\Omega}\left|D T_{k}(u)\right|^{p} d x \leq C(k+1) \quad \forall k>0,
$$

where $C$ is independent of $k$, then the cap $p$-quasi-continuous representative of $u$ is cap $_{p}$-quasi every where finite (see [22]).

From now on, $\Omega$ is assumed to be a bounded domain in $\mathbb{R}^{N}$ with $\partial \Omega$ of class $C^{1}$. Then, $\Omega$ is an extension domain (see [17]), so we can fix an open bounded subset $U_{\Omega}$ of $\mathbb{R}^{N}$ such that $\bar{\Omega} \subset U_{\Omega}$, and there exists a bounded linear operator $E: W^{1, p}(\Omega) \rightarrow W_{0}^{1, p}\left(U_{\Omega}\right)$ for which

(i) $E(u)=u$ a.e in $\Omega$ for each $u \in W^{1, p}(\Omega)$, 
(ii) $\|E(u)\|_{W_{0}^{1, p}\left(U_{\Omega}\right)} \leq C\|u\|_{W^{1, p}(\Omega)}$, where $C$ is a constant depending only on $p$ and $\Omega$.

We call $E(u)$ an extension of $u$ to $U_{\Omega}$. If $u \in W^{1, p}(\Omega), 1<p \leq \infty$, it is possible to give a pointwise definition of the trace $\tau(u)$ of $u$ on $\partial \Omega$ in the following way (see [34]), as $E(u) \in W_{0}^{1, p}\left(U_{\Omega}\right)$, every point of $U_{\Omega}$, except possibly a set of zero $p$-capacity, is a Lebesgue point of $E(u)$. Since $p>1$, the sets of zero $p$-capacity are of $\mathcal{H}^{N-1}$-measure zero and therefore $E(u)$ is defined $\mathcal{H}^{N-1}$-almost everywhere on $\partial \Omega$, so $\tau(u)=E(u)$ on $\partial \Omega$. This definition is independent of the open set $U_{\Omega}$ and also of the extension $E(u)$. We denote $\tau(u)$ by $u$ in the rest of the paper.

Lemma 2.1. Assume $\Omega$ is a bounded domain in $\mathbb{R}^{N}$ with $\partial \Omega$ of class $C^{1}$. Given $u \in \mathcal{T}^{1, p}(\Omega)$ there exists $\bar{u} \in \mathcal{T}_{0}^{1, p}\left(U_{\Omega}\right)$ such that

$$
T_{k}(\bar{u})=E\left[T_{k}(u)\right] \quad \text { for all } k>0 .
$$

Proof. To prove this result we need to recall the construction of the extension operator $E: W^{1, p}(\Omega) \rightarrow W_{0}^{1, p}\left(U_{\Omega}\right)$ given in [17]. For $x=\left(x_{1}, \ldots x_{N}\right) \in \mathbb{R}^{N}$, we write

$$
x=\left(x^{\prime}, x_{N}\right), \quad \text { with } x^{\prime} \in \mathbb{R}^{N-1}, \quad x^{\prime}=\left(x_{1}, \ldots, x_{N-1}\right),
$$

and we set

$$
\left|x^{\prime}\right|=\left(\sum_{i=1}^{N-1} x_{i}^{2}\right)^{\frac{1}{2}}
$$

We denote

$$
\begin{aligned}
Q & =\left\{\left(x^{\prime}, x_{N}\right) \in \mathbb{R}^{N-1} \times \mathbb{R}:\left|x^{\prime}\right|<1, \quad\left|x_{N}\right|<1\right\}, \\
Q_{+} & =\left\{\left(x^{\prime}, x_{N}\right) \in \mathbb{R}^{N-1} \times \mathbb{R}:\left|x^{\prime}\right|<1, \quad 0<x_{N}<1\right\}
\end{aligned}
$$

and

$$
Q_{0}=\left\{\left(x^{\prime}, x_{N}\right) \in \mathbb{R}^{N-1} \times \mathbb{R}:\left|x^{\prime}\right|<1, \quad x_{N}=0\right\} .
$$

Let $R: Q \rightarrow Q$ the reflection operator defined by

$$
R\left(x^{\prime}, x_{N}\right):= \begin{cases}\left(x^{\prime}, x_{N}\right) & \text { if } x_{N} \geq 0 \\ \left(x^{\prime},-x_{N}\right) & \text { if } x_{N}<0 .\end{cases}
$$

Since $\partial \Omega$ is of class $C^{1}$, there exist open sets $U_{i} \subset U_{\Omega}, i=1, \ldots, k$, such that

$$
\partial \Omega \subset \bigcup_{i=1}^{k} U_{i},
$$

and bijective functions $G_{i}: Q \rightarrow U_{i}$ such that $G_{i} \in C^{1}(\bar{Q}), G_{i}{ }^{-1} \in C^{1}\left(\overline{U_{i}}\right)$, $G_{i}\left(Q_{+}\right)=U_{i} \cap \Omega$ and $G_{i}\left(Q_{0}\right)=U_{i} \cap \partial \Omega$. Moreover, there exists a partition 
of unity $\left\{\theta_{i}\right\}_{i=0,1, \ldots, k}$ subordinate to $\partial \Omega$ and $U_{1}, \ldots U_{k}$ (see [17]), that is, $\theta_{i} \in$ $C^{\infty}\left(\mathbb{R}^{N}\right), 0 \leq \theta_{i} \leq 1$,

$$
\sum_{i=0}^{k} \theta_{i}=1 \text { in } \mathbb{R}^{N}
$$

$$
\left\{\begin{array}{l}
\operatorname{supp}\left(\theta_{i}\right) \text { is compact and } \operatorname{supp}\left(\theta_{i}\right) \subset U_{i}, \quad i=1, \ldots, k \\
\operatorname{supp}\left(\theta_{0}\right) \subset \mathbb{R}^{N} \backslash \partial \Omega, \text { and } \theta_{0 \mid \Omega} \in C_{c}^{\infty}(\Omega)
\end{array}\right.
$$

Given $w \in W^{1, p}(\Omega)$, for $x \in U_{\Omega}$, we have, setting $U_{0}=\Omega, F_{0}=I$ and $F_{i}=$ $G_{i} \circ R \circ G_{i}^{-1}, i=1,2, \ldots, k$,

$$
E(w)(x)=\sum_{i \in\{0,1, \ldots, k\}: x \in U_{i}} \theta_{i}(x) w\left(F_{i}(x)\right) .
$$

Fix $u \in \mathcal{T}^{1, p}(\Omega)$. First, observe that by (2.1), we have

$$
\left|E\left[T_{h}(u)\right](x)\right| \leq h \quad \forall h>0 .
$$

Let us prove that

$$
A:=\left\{x \in U_{\Omega}:\left|E\left[T_{h}(u)\right](x)\right| \geq h, \quad \forall h>0\right\}
$$

is an $\mathcal{L}^{N}$-null set. Obviously, if

$$
A_{\Omega}:=\left\{x \in \Omega:\left|E\left[T_{h}(u)\right](x)\right| \geq h, \quad \forall h>0\right\},
$$

we have $\mathcal{L}^{N}\left(A_{\Omega}\right)=0$. On the other hand, by (2.1) and (2.2), it is easy to see that

$$
A \backslash A_{\Omega} \subset \bigcup_{i=1}^{k}\left(G_{i} \circ R \circ G_{i}^{-1}\right)\left(A_{\Omega} \cap U_{i}\right)
$$

Consequently, $\mathcal{L}^{N}(A)=0$. Therefore, we can define $\mathcal{L}^{N}$-almost everywhere the function $\bar{u}: U_{\Omega} \rightarrow \mathbb{R}$ by

$$
\bar{u}(x):=E\left[T_{h}(u)\right](x), \quad \text { if } x \text { is such that }\left|E\left[T_{h}(u)\right](x)\right|<h,
$$

which is well defined by (2.2) and verifies the lemma.

Let $U$ be an open subset of $\mathbb{R}^{N}$. We set by $\mathcal{M}_{b}(U)$ the space of all Radon measures in $U$ with bounded total variation. We recall that for a measure $\mu \in$ $\mathcal{M}_{b}(U)$ and a Borel set $A \subset U$, the measure $\mu\llcorner A$ is defined by $(\mu\llcorner A)(B)=$ $\mu(B \cap A)$ for any Borel set $B \subset U$. If a measure $\mu \in \mathcal{M}_{b}(U)$ is such that $\mu=\mu\llcorner A$ for a certain Borel set $A$, the measure $\mu$ is said to be concentrated on $A$. For $\mu \in \mathcal{M}_{b}(U)$, we denote by $\mu^{+}, \mu^{-}$and $|\mu|$ the positive part, negative part and the total variation of the measure $\mu$, respectively. By $\mu=\mu_{a}+\mu_{s}$ we denote 
the Radon-Nikodym decomposition of $\mu$ relatively to $\mathcal{L}^{N}$. For simplicity, we write also $\mu_{a}$ for its density respect to $\mathcal{L}^{N}$, that is, for the function $f \in L^{1}(U)$ such that $\mu_{a}=f \mathcal{L}^{N}\llcorner U$.

Let $V$ be an open subset of $\mathbb{R}^{N}$. For a given measure $v \in \mathcal{M}_{b}(U)$ and a continuous function $f: U \rightarrow V$, the push-forward measure $f \# v$ is the Radon measure in $V$ defined by

$$
\langle f \# \nu, \varphi\rangle:=\int_{U} \varphi \circ f d \nu \quad \forall \varphi \in C_{c}(V) .
$$

We denote by $\mathcal{M}_{b}^{p}(U)$ the space of all diffuse Radon measures in $U$, i.e., measures which do not charge sets of zero $p$-capacity. In [13] it is proved that $\mu \in$ $\mathcal{M}_{b}(U)$ belongs to $\mathcal{M}_{b}^{p}(U)$ if and only if it belongs to $L^{1}(U)+W^{-1, p^{\prime}}(U)$, where $W^{-1, p^{\prime}}(U)=\left[W_{0}^{1, p}(U)\right]^{*}$. Moreover, if $u \in W^{1, p}(U)$ and $\mu \in \mathcal{M}_{b}^{p}(U)$, then $u$ is measurable with respect to $\mu$. If $u$ further belongs to $L^{\infty}(U)$, then $u$ belongs to $L^{\infty}(U, d \mu)$, hence to $L^{1}(U, d \mu)$.

We define

$$
\mathfrak{M}_{b}^{p}(\bar{\Omega}):=\left\{\mu \in \mathcal{M}_{b}^{p}\left(U_{\Omega}\right): \mu \text { is concentrated on } \bar{\Omega}\right\} .
$$

This definition is independent of the open set $U_{\Omega}$. Note that for $u \in W^{1, p}(\Omega) \cap$ $L^{\infty}(\Omega)$ and $\mu \in \mathfrak{M}_{b}^{p}(\bar{\Omega})$, we have

$$
\langle\mu, E(u)\rangle=\int_{\Omega} u d \mu+\int_{\partial \Omega} u d \mu ;
$$

on the other hand, there exists $f \in L^{1}\left(U_{\Omega}\right)$ and $F \in\left(L^{p^{\prime}}\left(U_{\Omega}\right)\right)^{N}$ such that $\mu=$ $f+\operatorname{div}(F)$, therefore, we also can write

$$
\langle\mu, E(u)\rangle=\int_{U_{\Omega}} f E(u) d x-\int_{U_{\Omega}} F \cdot D E(u) d x .
$$

Note that, if $f \in L^{1}(\Omega)$ and $g \in L^{1}(\partial \Omega)$ then $f \mathcal{L}^{N}\left\llcorner\Omega+g \mathcal{H}^{N-1}\llcorner\partial \Omega\right.$ is a diffuse measure concentrated in $\bar{\Omega}$. Now, if $p>N-k, 1 \leq k<N-1$, and $\mathbb{M}$ is a $k$-rectifiable subset of $\partial \Omega$, then $\mathcal{H}^{k}\llcorner\mathbb{M}$ is a diffuse measure concentrated in $\partial \Omega$ which is not an $L^{1}$ function in $\partial \Omega$ (see, [28, Theorem 2.26] or [34, Theorem 2.6.16]).

Let $\vartheta$ be a maximal monotone graph in $\mathbb{R} \times \mathbb{R}$. For $r \in \mathbb{N}$, the Yosida approximation $\vartheta_{r}$ of $\vartheta$ is given by $\vartheta_{r}=r\left(I-\left(I+\frac{1}{r} \vartheta\right)^{-1}\right)$. The function $\vartheta_{r}$ is maximal monotone and Lipschitz. We recall the definition of the main section $\vartheta^{0}$ of $\vartheta$

$$
\vartheta^{0}(s):= \begin{cases}\text { the element of minimal absolute value of } \vartheta(s) \text { if } \vartheta(s) \neq \emptyset, \\ +\infty & \text { if }[s,+\infty) \cap \operatorname{Dom}(\vartheta)=\emptyset \\ -\infty & \text { if }(-\infty, s] \cap \operatorname{Dom}(\vartheta)=\emptyset\end{cases}
$$


We have that $\left|\vartheta_{r}\right|$ is increasing in $r$, if $s \in \operatorname{Dom}(\vartheta), \vartheta_{r}(s) \rightarrow \vartheta^{0}(s)$ as $r \rightarrow+\infty$, and if $s \notin \operatorname{Dom}(\theta),\left|\vartheta_{r}(s)\right| \rightarrow+\infty$ as $r \rightarrow+\infty$.

We set

$$
\vartheta(r+):=\inf \vartheta(] r,+\infty[), \quad \vartheta(r-):=\sup \vartheta(]-\infty, r[)
$$

for $r \in \mathbb{R}$, where we use the conventions inf $\emptyset=+\infty$ and $\sup \emptyset=-\infty$. It is easy to see that

$$
\vartheta(r)=[\vartheta(r-), \vartheta(r+)] \cap \mathbb{R} \text { for } r \in \mathbb{R} \text {. }
$$

Moreover,

$$
J(\vartheta):=\{\theta \in \operatorname{Dom}(\vartheta): \vartheta(r-)<\vartheta(r+)\}
$$

is a countable set.

We shall denote $\vartheta_{-}:=\inf \operatorname{Ran}(\vartheta)$ and $\vartheta_{+}:=\sup \operatorname{Ran}(\vartheta)$. If $0 \in \operatorname{Dom}(\vartheta)$, $j_{\vartheta}(r)=\int_{0}^{r} \vartheta^{0}(s) d s$ defines a convex lower semi-continuous function such that $\vartheta=\partial j_{\vartheta}$. If $j_{\vartheta}^{*}$ is the Legendre transformation of $j_{\vartheta}$ then $\vartheta^{-1}=\partial j_{\vartheta}^{*}$.

To finish these preliminaries, let us recall some of the results obtained in [2] for the case of integrable functions that will be used afterward.

We set

$V^{1, p}(\Omega):=\left\{\phi \in L^{1}(\Omega): \exists M>0\right.$ such that $\left.\int_{\Omega}|\phi v| \leq M\|v\|_{W^{1, p}(\Omega)} \forall v \in W^{1, p}(\Omega)\right\}$

and

$$
\begin{aligned}
& V^{1, p}(\partial \Omega) \\
& :=\left\{\psi \in L^{1}(\partial \Omega): \exists M>0 \text { such that } \int_{\partial \Omega}|\psi v| \leq M\|v\|_{W^{1, p}(\Omega)} \forall v \in W^{1, p}(\Omega)\right\} .
\end{aligned}
$$

$V^{1, p}(\Omega)$ is a Banach space endowed with the norm

$$
\|\phi\|_{V^{1, p}(\Omega)}:=\inf \left\{M>0: \int_{\Omega}|\phi v| \leq M\|v\|_{W^{1, p}(\Omega)} \forall v \in W^{1, p}(\Omega)\right\},
$$

and $V^{1, p}(\partial \Omega)$ is a Banach space endowed with the norm

$$
\|\psi\|_{V^{1, p}(\partial \Omega)}:=\inf \left\{M>0: \int_{\partial \Omega}|\psi v| \leq M\|v\|_{W^{1, p}(\Omega)} \forall v \in W^{1, p}(\Omega)\right\} .
$$

Observe that, Sobolev embedding and Trace theorems imply, for $1 \leq p<N$,

$$
L^{p^{\prime}}(\Omega) \subset L^{(N p /(N-p))^{\prime}}(\Omega) \subset V^{1, p}(\Omega)
$$

and

$$
L^{p^{\prime}}(\partial \Omega) \subset L^{((N-1) p /(N-p))^{\prime}}(\partial \Omega) \subset V^{1, p}(\partial \Omega) .
$$

For the maximal monotone graphs $\gamma$ and $\beta$, we shall denote

$$
\mathcal{R}_{\gamma, \beta}^{+}:=\gamma_{+} \mathcal{L}^{N}(\Omega)+\beta_{+} \mathcal{H}^{N-1}(\partial \Omega), \quad \mathcal{R}_{\gamma, \beta}^{-}:=\gamma_{-} \mathcal{L}^{N}(\Omega)+\beta_{-} \mathcal{H}^{N-1}(\partial \Omega) .
$$

We will suppose $\mathcal{R}_{\gamma, \beta}^{-}<\mathcal{R}_{\gamma, \beta}^{+}$and we will write $\left.\mathcal{R}_{\gamma, \beta}:=\right] \mathcal{R}_{\gamma, \beta}^{-}, \mathcal{R}_{\gamma, \beta}^{+}[$. 
Theorem 2.2 ([2]). Assume $\operatorname{Dom}(\beta)=\mathbb{R}$. For any $\phi \in V^{1, p}(\Omega)$ such that $\int_{\Omega} \phi \in$ $\mathcal{R}_{\gamma, \beta}$, there exists a weak solution $[u, z, w] \in W^{1, p}(\Omega) \times V^{1, p}(\Omega) \times V^{1, p}(\partial \Omega)$ of $\left(S_{\phi, 0}^{\gamma, \beta}\right)$, that is,

$$
\int_{\Omega} \mathbf{a}(x, D u) \cdot D v+\int_{\Omega} z v+\int_{\partial \Omega} w v=\int_{\Omega} \phi v, \quad \forall v \in W^{1, p}(\Omega) .
$$

Moreover,

$$
\left\|z^{ \pm}\right\|_{L^{1}(\Omega)}+\left\|w^{ \pm}\right\|_{L^{1}(\partial \Omega)} \leq\left\|\phi^{ \pm}\right\|_{L^{1}(\Omega)} .
$$

Theorem 2.3 ([2]). For any $\phi \in V^{1, p}(\Omega)$ there exists a weak solution $[u, z] \in$ $W_{0}^{1, p}(\Omega) \times V^{1, p}(\Omega)$ of $\left(S_{\phi, 0}^{\gamma, D}\right)$, that is,

$$
\int_{\Omega} \mathbf{a}(x, D u) \cdot D v+\int_{\Omega} z v=\int_{\Omega} \phi v, \quad \forall v \in W_{0}^{1, p}(\Omega),
$$

and

$$
\left\|z^{ \pm}\right\|_{L^{1}(\Omega)} \leq\left\|\phi^{ \pm}\right\|_{L^{1}(\Omega)}
$$

\section{The concepts of solution}

We introduce the following concepts of solution for problem $\left(S_{\mu_{1}, \mu_{2}}^{\gamma, \beta}\right)$.

Definition 3.1. Let $\mu_{1}, \mu_{2}$ measures, $\mu_{1}=\mu_{1}\left\llcorner\Omega\right.$ and $\mu_{2}=\mu_{2}\llcorner\partial \Omega$, such that $\mu_{1}+\mu_{2} \in \mathfrak{M}_{b}^{p}(\bar{\Omega})$. A triple of functions $[u, z, w] \in W^{1, p}(\Omega) \times L^{1}(\Omega) \times L^{1}(\partial \Omega)$ is a weak solution of problem $\left(S_{\mu_{1}, \mu_{2}}^{\gamma, \beta}\right)$ if $z(x) \in \gamma(u(x))$ a.e. in $\Omega, w(x) \in$ $\beta(u(x))$ a.e. in $\partial \Omega$ and

$$
\int_{\Omega} \mathbf{a}(x, D u) \cdot D v d x+\int_{\Omega} z v d x+\int_{\partial \Omega} w v d \mathcal{H}^{N-1}=\int_{\Omega} v d \mu_{1}+\int_{\partial \Omega} v d \mu_{2}
$$

for all $v \in W^{1, p}(\Omega) \cap L^{\infty}(\Omega)$.

Let us remark that the fact of being the weak solution in the energy space forces the measure $\mu_{1}+\mu_{2}$ to belong to a dual space (see Theorem 4.5).

As we pointed out in the introduction, for this type of problems, the concept of weak solution is not enough in order to get uniqueness. It is necessary to find some extra conditions on the distributional solutions in order to ensure both existence and uniqueness. This was done, introducing the concepts of entropy and renormalized solutions (see [6]). For our problem these concepts are the following.

Definition 3.2. Let $\mu_{1}, \mu_{2}$ measures, $\mu_{1}=\mu_{1}\left\llcorner\Omega\right.$ and $\mu_{2}=\mu_{2}\llcorner\partial \Omega$, such that $\mu_{1}+\mu_{2} \in \mathfrak{M}_{b}^{p}(\bar{\Omega})$. A triple of functions $[u, z, w] \in \mathcal{T}_{\text {tr }}^{1, p}(\Omega) \times L^{1}(\Omega) \times L^{1}(\partial \Omega)$ 
is an entropy solution of problem $\left(S_{\mu_{1}, \mu_{2}}^{\gamma, \beta}\right)$ if $z(x) \in \gamma(u(x))$ a.e. in $\Omega, w(x) \in$ $\beta(u(x))$ a.e. in $\partial \Omega$ and

$$
\begin{aligned}
& \int_{\Omega} \mathbf{a}(x, D u) \cdot D T_{k}(u-v) d x+\int_{\Omega} z T_{k}(u-v) d x+\int_{\partial \Omega} w T_{k}(u-v) d \mathcal{H}^{N-1} \\
& \leq \int_{\Omega} T_{k}(u-v) d \mu_{1}+\int_{\partial \Omega} T_{k}(u-v) d \mu_{2} \quad \forall k>0,
\end{aligned}
$$

for all $v \in W^{1, p}(\Omega) \cap L^{\infty}(\Omega)$.

Definition 3.3. Let $\mu_{1}, \mu_{2}$ measures, $\mu_{1}=\mu_{1}\left\llcorner\Omega\right.$ and $\mu_{2}=\mu_{2}\llcorner\partial \Omega$, such that $\mu_{1}+\mu_{2} \in \mathfrak{M}_{b}^{p}(\bar{\Omega})$. A triple of functions $[u, z, w] \in \mathcal{T}_{\text {tr }}^{1, p}(\Omega) \times L^{1}(\Omega) \times L^{1}(\partial \Omega)$ is a renormalized solution of problem $\left(S_{\mu_{1}, \mu_{2}}^{\gamma, \beta}\right)$ if $z(x) \in \gamma(u(x))$ a.e. in $\Omega, w(x) \in$ $\beta(u(x))$ a.e. in $\partial \Omega$, and the following conditions hold

(a) for every $h \in W^{1, \infty}(\mathbb{R})$ with compact support we have

$$
\begin{aligned}
& \int_{\Omega} \mathbf{a}(x, D u) \cdot D u h^{\prime}(u) \varphi d x+\int_{\Omega} \mathbf{a}(x, D u) \cdot D \varphi h(u) d x \\
& \quad+\int_{\Omega} z h(u) \varphi d x+\int_{\partial \Omega} w h(u) \varphi d \mathcal{H}^{N-1} \\
& =\int_{\Omega} h(u) \varphi d \mu_{1}+\int_{\partial \Omega} h(u) \varphi d \mu_{2} \quad \forall k>0,
\end{aligned}
$$

(b)

for all $\varphi \in W^{1, p}(\Omega) \cap L^{\infty}(\Omega)$ such that $h(u) \varphi \in W^{1, p}(\Omega)$,

$$
\lim _{n \rightarrow+\infty} \int_{\{n \leq|u| \leq n+1\}} \mathbf{a}(x, D u) \cdot D u d x=0 .
$$

Remark 3.4. Every term in (3.2) is well defined. This is clear for the right hand side since $h(u) \varphi$ belongs to $L^{\infty}\left(\bar{\Omega}, \mu_{1}+\mu_{2}\right)$, and thus to $L^{1}\left(\bar{\Omega}, \mu_{1}+\mu_{2}\right)$. On the other hand, since $\operatorname{supp}(h) \subset[-k, k]$ for some $k>0$, the two first terms of the left hand side can be written as

$$
\int_{\Omega} \mathbf{a}\left(x, D T_{k}(u)\right) \cdot D T_{k}(u) h^{\prime}(u) \varphi d x+\int_{\Omega} \mathbf{a}\left(x, D T_{k}(u)\right) \cdot D \varphi h(u) d x,
$$

and both integrals are well defined in view of $\left(H_{2}\right)$, since both $\varphi$ and $T_{k}(u)$ belong to $W^{1, p}(\Omega)$. Moreover, it is not difficult to see that the product $D T_{k}(u) h^{\prime}(u)$ coincides with the gradient of the composite function $h(u)=h\left(T_{k}(u)\right)$ almost everywhere (see [14]).

In the next result we will see that entropy and renormalized solutions coincide.

Theorem 3.5. Let $\mu_{1}, \mu_{2}$ measures, $\mu_{1}=\mu_{1}\left\llcorner\Omega\right.$ and $\mu_{2}=\mu_{2}\llcorner\partial \Omega$, such that $\mu_{1}+\mu_{2} \in \mathfrak{M}_{b}^{p}(\bar{\Omega})$. Then, $[u, z, w]$ is an entropy solution of problem $\left(S_{\mu_{1}, \mu_{2}}^{\gamma, \beta}\right)$ if and only if $[u, z, w]$ is a renormalized solution of problem $\left(S_{\mu_{1}, \mu_{2}}^{\gamma, \beta}\right)$. 
To prove the above theorem we firstly show the following lemma:

Lemma 3.6. Let $\mu_{1}, \mu_{2}$ measures, $\mu_{1}=\mu_{1}\left\llcorner\Omega\right.$ and $\mu_{2}=\mu_{2}\llcorner\partial \Omega$, such that $\mu_{1}+\mu_{2} \in \mathfrak{M}_{b}^{p}(\bar{\Omega})$. Let $[u, z, w]$ be an entropy solution of problem $\left(S_{\mu_{1}, \mu_{2}}^{\gamma, \beta}\right)$. Then,

$$
\lim _{h \rightarrow+\infty} \int_{\{x \in \Omega: h<|u(x)|<h+k\}}|D u|^{p}=0, \quad \forall k>0 .
$$

Proof. Let us write $\mu_{1}+\mu_{2}=f+\operatorname{div} F$ in $\mathcal{D}^{\prime}\left(U_{\Omega}\right), f \in L^{1}\left(U_{\Omega}\right)$, and $F \in$ $\left(L^{p^{\prime}}\left(U_{\Omega}\right)\right)^{N}$. First, let us see that for all $h>0$,

$$
\begin{aligned}
& \int_{\{x \in \Omega: h<|u(x)|<h+k\}}|D u|^{p} \\
& \leq M\left(k \int_{\left\{x \in U_{\Omega}:\left|v_{h}(x)\right| \geq h\right\}}|f|+\int_{\left\{x \in U_{\Omega}:\left|v_{h}(x)\right|>h\right\}}|F|^{p^{\prime}}+\int_{\Omega}\left|T_{k}\left(u-T_{h}(u)\right)\right|^{p}\right),
\end{aligned}
$$

where $v_{h}=E\left[T_{k+h}\right](u)$ is the extension of $T_{k+h}(u)$ to $W_{0}^{1, p}\left(U_{\Omega}\right)$ and the constant $M$ is independent of $h$ and $k$. Indeed, taking $T_{h}(u)$ as test function in (3.1), since $E\left[T_{k}\left(u-T_{h}(u)\right)\right]=T_{k}\left(v_{h}-T_{h}\left(v_{h}\right)\right)$, we have

$$
\begin{aligned}
& \int_{\Omega} \mathbf{a}(x, D u) \cdot D T_{k}\left(u-T_{h}(u)\right)+\int_{\Omega} z T_{k}\left(u-T_{h}(u)\right)+\int_{\partial \Omega} w T_{k}\left(u-T_{h}(u)\right) \\
& \leq \int_{U_{\Omega}} f T_{k}\left(v_{h}-T_{h}\left(v_{h}\right)\right)-\int_{U_{\Omega}} D T_{k}\left(v_{h}-T_{h}\left(v_{h}\right)\right) \cdot F .
\end{aligned}
$$

Now, since

$$
\int_{U_{\Omega}}\left|D T_{k}\left(v_{h}-T_{h}\left(v_{h}\right)\right)\right|^{p} \leq C\left(\int_{\Omega}\left|D T_{k}\left(u-T_{h}(u)\right)\right|^{p}+\int_{\Omega}\left|T_{k}\left(u-T_{h}(u)\right)\right|^{p}\right),
$$

using Young's inequality, we get that there exists a constant $M_{1}$ independent of $h$ and $k$ such that

$$
\begin{aligned}
& \left|\int_{U_{\Omega}} D T_{k}\left(v_{h}-T_{h}\left(v_{h}\right)\right) \cdot F\right| \leq M_{1} \int_{\left\{\left|v_{h}\right|>h\right\}}|F|^{p^{\prime}}+\frac{\Lambda}{2 C} \int_{U_{\Omega}}\left|D T_{k}\left(v_{h}-T_{h}\left(v_{h}\right)\right)\right|^{p} \\
& \quad \leq M_{1} \int_{\left\{\left|v_{h}\right|>h\right\}}|F|^{p^{\prime}}+\frac{\Lambda}{2}\left(\int_{\Omega}\left|D T_{k}\left(u-T_{h}(u)\right)\right|^{p}+\int_{\Omega}\left|T_{k}\left(u-T_{h}(u)\right)\right|^{p}\right) .
\end{aligned}
$$

Then, by $\left(H_{1}\right)$ and the positivity of the second and third terms in (3.6), it follows (3.5).

Let $\bar{u}$ be the function obtained by Lemma 2.1. Since $v_{h}=T_{h+k}(\bar{u})$, we have $\left\{\left|v_{h}\right| \geq h\right\} \subset\{|\bar{u}| \geq h\}$, which implies that

$$
\lim _{h \rightarrow+\infty} \mathcal{L}^{N}\left(\left\{\left|v_{h}\right| \geq h\right\}\right)=0 .
$$

Consequently, from (3.5), (3.4) is deduced. 
Proof of Theorem 3.5. Let $\mu_{1}, \mu_{2}$ measures, $\mu_{1}=\mu_{1} L \Omega$ and $\mu_{2}=\mu_{2} L \partial \Omega$, such that $\mu=\mu_{1}+\mu_{2} \in \mathfrak{M}_{b}^{p}(\bar{\Omega})$.

Assume that $[u, z, w]$ is a renormalized solution. Fix $v \in W^{1, p}(\Omega) \cap L^{\infty}(\Omega)$. Let $h_{n}(r)=\inf \left(1,(n+1-|r|)^{+}\right)$and $\varphi=T_{k}(u-v)$. Then,

$$
\begin{gathered}
\int_{\Omega} h_{n}^{\prime}(u) \mathbf{a}(x, D u) \cdot D u T_{k}(u-v) d x+\int_{\Omega} \mathbf{a}(x, D u) \cdot D T_{k}(u-v) h_{n}(u) d x \\
\quad+\int_{\Omega} z h_{n}(u) T_{k}(u-v) d x+\int_{\partial \Omega} w h_{n}(u) T_{k}(u-v) d \mathcal{H}^{N-1} \\
=\int_{\Omega} h_{n}(u) T_{k}(u-v) d \mu_{1}+\int_{\partial \Omega} h_{n}(u) T_{k}(u-v) d \mu_{2} \quad \forall k>0 .
\end{gathered}
$$

Since, for $n$ large enough we have

$$
D\left(h_{n}(u) T_{k}(u-v)\right)=D T_{k}(u-v)+h_{n}^{\prime}(u) T_{k}(u-v) D u,
$$

by (3.3) we have

$$
\lim _{n \rightarrow+\infty} \int_{\Omega} h_{n}^{\prime}(u) \mathbf{a}(x, D u) \cdot D u T_{k}(u-v)=0 .
$$

Moreover

$$
\lim _{n \rightarrow+\infty} \int_{\bar{\Omega}} h_{n}(u) T_{k}(u-v) d \mu=\int_{\bar{\Omega}} T_{k}(u-v) d \mu
$$

and, we deduce that $u$ is an entropy solution.

Assume now that $[u, z, w]$ is an entropy solution of problem $\left(S_{\mu_{1}, \mu_{2}}^{\gamma, \beta}\right)$. By Lemma 3.6, (3.3) holds. Let $h \in W^{1, \infty}(\mathbb{R})$ with compact support and $\varphi \in W^{1, p}(\Omega) \cap$ $L^{\infty}(\Omega)$ such that $h(u) \varphi \in W^{1, p}(\Omega)$. Take now as a test function in (3.1) $v=$ $T_{l}(u)-h(u) \varphi$ and $k=\|h(u) \varphi\|_{L^{\infty}(\Omega)}$. For $l$ large enough such that $h(r)=0$ if $|r| \geq l$, we have

$$
D T_{k}(u-v)=D(h(u) \varphi) \chi_{[|u| \leq l]}+D u \chi_{[l \leq|u| \leq l+k]},
$$

moreover, $T_{k}(u-v) \rightarrow T_{k}(h(u) \varphi)=h(u) \varphi$ as $l \rightarrow \infty$. So, by letting $l \rightarrow \infty$, by (3.4), we deduce that, for every $h \in W^{1, \infty}(\mathbb{R})$ with compact support

$$
\begin{gathered}
\int_{\Omega} \mathbf{a}(x, D u) \cdot D u h^{\prime}(u) \varphi d x+\int_{\Omega} \mathbf{a}(x, D u) \cdot D \varphi h(u) d x \\
\quad+\int_{\Omega} z h(u) \varphi d x+\int_{\partial \Omega} w h(u) \varphi d \mathcal{H}^{N-1} \\
\leq \int_{\Omega} h(u) \varphi d \mu_{1}+\int_{\partial \Omega} h(u) \varphi d \mu_{2} \quad \forall k>0 .
\end{gathered}
$$

Interchanging $\varphi$ and $-\varphi$, the equality is obtained. 
Remark 3.7. Assume that $[u, z, w]$ is an entropy solution of problem $\left(S_{\mu_{1}, \mu_{2}}^{\gamma, \beta}\right)$. If we take $v=T_{h}(u) \pm 1$ as test functions in (3.1) and let $h$ go to $+\infty$, we get that

$$
\int_{\Omega} z+\int_{\partial \Omega} w=\mu_{1}(\Omega)+\mu_{2}(\partial \Omega) .
$$

Then, since $z(x) \in \gamma(u(x))$ a.e. in $\Omega$ and $w(x) \in \beta(u(x))$ a.e. in $\partial \Omega$, necessarily $\mu_{1}$ and $\mu_{2}$ must satisfy

$$
\mathcal{R}_{\gamma, \beta}^{-} \leq \mu_{1}(\Omega)+\mu_{2}(\partial \Omega) \leq \mathcal{R}_{\gamma, \beta}^{+}
$$

\section{The Existence and Uniqueness Results}

For weak solutions a contraction principle is proved in Theorem 4.5. With respect to uniqueness for entropy solutions we have the following general result (see [13] for the homogeneous Dirichlet problem).

Theorem 4.1. Let $\mu_{1}, \mu_{2}$ measures, $\mu_{1}=\mu_{1}\left\llcorner\Omega\right.$ and $\mu_{2}=\mu_{2}\llcorner\partial \Omega$, such that $\mu_{1}+\mu_{2} \in \mathfrak{M}_{b}^{p}(\bar{\Omega})$. Let $\left[u_{1}, z_{1}, w_{1}\right]$ and $\left[u_{2}, z_{2}, w_{2}\right]$ be entropy solutions of problem $\left(S_{\mu_{1}, \mu_{2}}^{\gamma, \beta}\right)$. Then, there exists a constant $c \in \mathbb{R}$ such that

$$
\begin{gathered}
u_{1}-u_{2}=c \quad \text { a.e. in } \Omega, \\
z_{1}-z_{2}=0 \quad \text { a.e. in } \Omega . \\
w_{1}-w_{2}=0 \quad \text { a.e. in } \partial \Omega .
\end{gathered}
$$

Moreover, if $c \neq 0$, there exists a constant $k \in \mathbb{R}$ such that $z_{1}=z_{2}=k$.

Proof. Let us write $\mu_{1}+\mu_{2}=f+\operatorname{div} F$ in $\mathcal{D}^{\prime}\left(U_{\Omega}\right), f \in L^{1}\left(U_{\Omega}\right), F \in\left(L^{p^{\prime}}\left(U_{\Omega}\right)\right)^{N}$. For every $h>0$, we have that

$$
\begin{aligned}
& \int_{\Omega} \mathbf{a}\left(x, D u_{1}\right) \cdot D T_{k}\left(u_{1}-T_{h}\left(u_{2}\right)\right)+\int_{\Omega} z_{1} T_{k}\left(u_{1}-T_{h}\left(u_{2}\right)\right)+\int_{\partial \Omega} w_{1} T_{k}\left(u_{1}-T_{h}\left(u_{2}\right)\right) \\
& \leq \int_{U_{\Omega}} f T_{k}\left(v_{1}-T_{h}\left(v_{2}\right)\right)-\int_{U_{\Omega}} F \cdot D T_{k}\left(v_{1}-T_{h}\left(v_{2}\right)\right)
\end{aligned}
$$

and

$$
\begin{aligned}
& \int_{\Omega} \mathbf{a}\left(x, D u_{2}\right) \cdot D T_{k}\left(u_{2}-T_{h}\left(u_{1}\right)\right)+\int_{\Omega} z_{2} T_{k}\left(u_{2}-T_{h}\left(u_{1}\right)\right)+\int_{\partial \Omega} w_{2} T_{k}\left(u_{2}-T_{h}\left(u_{1}\right)\right) \\
& \leq \int_{U_{\Omega}} f T_{k}\left(v_{2}-T_{h}\left(v_{1}\right)\right)-\int_{U_{\Omega}} F \cdot D T_{k}\left(v_{2}-T_{h}\left(v_{1}\right)\right)
\end{aligned}
$$


where $v_{i}:=E\left[T_{k+h}\left(u_{i}\right)\right], i=1,2$, is the extension of $T_{k+h}\left(u_{i}\right)$ to $W_{0}^{1, p}\left(U_{\Omega}\right)$. Adding both inequalities and taking limits when $h$ goes to $\infty$, on account of the monotonicity of $\gamma$ and $\beta$, if

$$
I_{h, k}:=\int_{\Omega} \mathbf{a}\left(x, D u_{1}\right) \cdot D T_{k}\left(u_{1}-T_{h}\left(u_{2}\right)\right)+\int_{\Omega} \mathbf{a}\left(x, D u_{2}\right) \cdot D T_{k}\left(u_{2}-T_{h}\left(u_{1}\right)\right),
$$

we get

$$
\begin{aligned}
& \limsup _{h \rightarrow \infty} I_{h, k}+\int_{\Omega}\left(z_{1}-z_{2}\right) T_{k}\left(u_{1}-u_{2}\right)+\int_{\partial \Omega}\left(w_{1}-w_{2}\right) T_{k}\left(u_{1}-u_{2}\right) \\
& \quad \leq \limsup _{h \rightarrow \infty}\left[-\int_{U_{\Omega}} F \cdot D\left(T_{k}\left(v_{1}-T_{h}\left(v_{2}\right)\right)+T_{k}\left(v_{2}-T_{h}\left(v_{1}\right)\right)\right)\right] .
\end{aligned}
$$

Let us see that

$$
\limsup _{h \rightarrow \infty}\left[-\int_{U_{\Omega}} F \cdot D\left(T_{k}\left(v_{1}-T_{h}\left(v_{2}\right)\right)+T_{k}\left(v_{2}-T_{h}\left(v_{1}\right)\right)\right)\right]=0 .
$$

In fact,

$$
\begin{aligned}
& -\int_{U_{\Omega}} F \cdot D\left(T_{k}\left(v_{1}-T_{h}\left(v_{2}\right)\right)+T_{k}\left(v_{2}-T_{h}\left(v_{1}\right)\right)\right) \\
= & -\int_{\left\{\left|v_{1}\right|<h,\left|v_{2}\right|>h\right\}} F \cdot D\left(T_{k}\left(v_{1}-T_{h}\left(v_{2}\right)\right)+T_{k}\left(v_{2}-T_{h}\left(v_{1}\right)\right)\right) \\
& -\int_{\left\{\left|v_{1}\right|>h,\left|v_{2}\right|<h\right\}} F \cdot D\left(T_{k}\left(v_{1}-T_{h}\left(v_{2}\right)\right)+T_{k}\left(v_{2}-T_{h}\left(v_{1}\right)\right)\right) \\
& -\int_{\left\{\left|v_{1}\right|>h,\left|v_{2}\right|>h\right\}} F \cdot D\left(T_{k}\left(v_{1}-T_{h}\left(v_{2}\right)\right)+T_{k}\left(v_{2}-T_{h}\left(v_{1}\right)\right)\right) \\
= & -\int_{\left\{\left|v_{1}\right|<h,\left|v_{2}\right|>h,\left|v_{1}-h \operatorname{sign}\left(v_{2}\right)\right|<k\right\}} F \cdot D v_{1} \\
& -\int_{\left\{\left|v_{1}\right|<h,\left|v_{2}\right|>h,\left|v_{2}-v_{1}\right|<k\right\}} F \cdot D\left(v_{2}-v_{1}\right) \\
& -\int_{\left\{\left|v_{1}\right|>h,\left|v_{2}\right|<h,\left|v_{1}-v_{2}\right|<k\right\}} F \cdot D\left(v_{1}-v_{2}\right) \\
& -\int_{\left\{\left|v_{1}\right|>h,\left|v_{2}\right|<h,\left|v_{2}-h \operatorname{sign}\left(v_{1}\right)\right|<k\right\}} F \cdot D v_{2} \\
& -\int_{\left\{\left|v_{1}\right|>h,\left|v_{2}\right|>h,\left|v_{1}-h \operatorname{sign}\left(v_{2}\right)\right|<k\right\}} F \cdot D v_{1} \\
& -\int_{\left\{\left|v_{1}\right|>h,\left|v_{2}\right|>h,\left|v_{2}-h \operatorname{sign}\left(v_{1}\right)\right|<k\right\}} F \cdot D v_{2} .
\end{aligned}
$$


Now, by Hölder's inequality

$$
\begin{aligned}
& \left|\int_{\left\{\left|v_{1}\right|<h,\left|v_{2}\right|>h,\left|v_{1}-h \operatorname{sign}\left(v_{2}\right)\right|<k\right\}} F \cdot D v_{1}\right| \leq\left|\int_{\left\{h-k<\left|v_{1}\right|<h,\right\}} F \cdot D v_{1}\right| \\
& =\left|\int_{U_{\Omega}} F \cdot D T_{k}\left(v_{1}-T_{h-k}\left(v_{1}\right)\right)\right| \leq\left(\int_{U_{\Omega}}|F|^{p^{\prime}}\right)^{\frac{1}{p^{\prime}}}\left(\int_{U_{\Omega}}\left|D T_{k}\left(v_{1}-T_{h-k}\left(v_{1}\right)\right)\right|^{p}\right)^{\frac{1}{p}} \\
& \leq C\left(\int_{U_{\Omega}}|F|^{p^{\prime}}\right)^{\frac{1}{p^{\prime}}}\left(\int_{\Omega}\left|D T_{k}\left(u_{1}-T_{h-k}\left(u_{1}\right)\right)\right|^{p}+\int_{\Omega}\left|T_{k}\left(u_{1}-T_{h-k}\left(u_{1}\right)\right)\right|^{p}\right)^{\frac{1}{p}} \\
& \leq C\left(\int_{U_{\Omega}}|F|^{p^{\prime}}\right)^{\frac{1}{p^{\prime}}}\left(\int_{\left\{h-k<\left|u_{1}\right|<h\right\}}\left|D u_{1}\right|^{p}+\int_{\Omega}\left|T_{k}\left(u_{1}-T_{h-k}\left(u_{1}\right)\right)\right|^{p}\right)^{\frac{1}{p}} .
\end{aligned}
$$

Hence, by Lemma 3.6, we obtain that

$$
\lim _{h \rightarrow+\infty} \int_{\left\{\left|v_{1}\right|<h,\left|v_{2}\right|>h,\left|v_{1}-h \operatorname{sign}\left(v_{2}\right)\right|<k\right\}} F \cdot D v_{1}=0 .
$$

Similarly, it can be proved that the five other terms of the last equality converge to 0 as $h \rightarrow+\infty$. Consequently, (4.2) holds. Now, from (4.1) and (4.2), we get

$$
\limsup _{h \rightarrow \infty} I_{h, k} \leq-\int_{\Omega}\left(z_{1}-z_{2}\right) T_{k}\left(u_{1}-u_{2}\right)-\int_{\partial \Omega}\left(w_{1}-w_{2}\right) T_{k}\left(u_{1}-u_{2}\right) \leq 0 .
$$

Let us see that

$$
\liminf _{h \rightarrow \infty} I_{h, k} \geq 0 \quad \text { for any } k
$$

To prove this, we split

$$
I_{h, k}=I_{h, k}^{1}+I_{h, k}^{2}+I_{h, k}^{3}+I_{h, k}^{4}
$$

where

$$
\begin{aligned}
I_{h, k}^{1}:= & \int_{\left\{\left|u_{1}\right|<h,\left|u_{2}\right|<h\right\}}\left(\mathbf{a}\left(x, D u_{1}\right)-\mathbf{a}\left(x, D u_{2}\right)\right) \cdot D T_{k}\left(u_{1}-u_{2}\right), \\
I_{h, k}^{2}:= & \int_{\left\{\left|u_{1}\right|<h,\left|u_{2}\right| \geq h\right\}} \mathbf{a}\left(x, D u_{1}\right) \cdot D T_{k}\left(u_{1}-h \operatorname{sign}\left(u_{2}\right)\right) \\
& +\int_{\left\{\left|u_{1}\right|<h,\left|u_{2}\right| \geq h\right\}} \mathbf{a}\left(x, D u_{2}\right) \cdot D T_{k}\left(u_{2}-u_{1}\right) \\
\geq & \int_{\left\{\left|u_{1}\right|<h,\left|u_{2}\right| \geq h\right\}} \mathbf{a}\left(x, D u_{2}\right) \cdot D T_{k}\left(u_{2}-u_{1}\right), \\
I_{h, k}^{3}:= & \int_{\left\{\left|u_{1}\right| \geq h,\left|u_{2}\right|<h\right\}} \mathbf{a}\left(x, D u_{1}\right) \cdot D T_{k}\left(u_{1}-u_{2}\right) \\
& +\int_{\left\{\left|u_{1}\right| \geq h,\left|u_{2}\right|<h\right\}} \mathbf{a}\left(x, D u_{2}\right) \cdot D T_{k}\left(u_{2}-h \operatorname{sign}\left(u_{1}\right)\right) \\
\geq & \int_{\left\{\left|u_{1}\right| \geq h,\left|u_{2}\right|<h\right\}} \mathbf{a}\left(x, D u_{1}\right) \cdot D T_{k}\left(u_{1}-u_{2}\right)
\end{aligned}
$$


and

$$
\begin{aligned}
& I_{h, k}^{4}:=\int_{\left\{\left|u_{1}\right| \geq h,\left|u_{2}\right| \geq h\right\}} \mathbf{a}\left(x, D u_{1}\right) \cdot D T_{k}\left(u_{1}-h \operatorname{sign}\left(u_{2}\right)\right) \\
& +\int_{\left\{\left|u_{1}\right| \geq h,\left|u_{2}\right| \geq h\right\}} \mathbf{a}\left(x, D u_{2}\right) \cdot D T_{k}\left(u_{2}-h \operatorname{sign}\left(u_{1}\right)\right) \geq 0 .
\end{aligned}
$$

Combining the above estimates we obtain

$$
I_{h, k} \geq I_{h, k}^{1}+L_{h, k}^{1}+L_{h, k}^{2},
$$

where

$$
\begin{aligned}
L_{h, k}^{1} & :=\int_{\left\{\left|u_{1}\right|<h,\left|u_{2}\right| \geq h\right\}} \mathbf{a}\left(x, D u_{2}\right) \cdot D T_{k}\left(u_{2}-u_{1}\right), \\
L_{h, k}^{2} & :=\int_{\left\{\left|u_{1}\right| \geq h,\left|u_{2}\right|<h\right\}} \mathbf{a}\left(x, D u_{1}\right) \cdot D T_{k}\left(u_{1}-u_{2}\right)
\end{aligned}
$$

and $I_{h, k}^{1}$ is nonnegative and nondecreasing in $h$. If we set

$$
C(h, k):=\left\{h<\left|u_{1}\right|<k+h\right\} \cap\left\{h-k<\left|u_{2}\right|<h\right\},
$$

we have

$$
\begin{aligned}
\left|L_{h, k}^{2}\right| & \leq \int_{\left\{\left|u_{1}-u_{2}\right|<k,\left|u_{1}\right| \geq h,\left|u_{2}\right|<h\right\}}\left|\mathbf{a}\left(x, D u_{1}\right) \cdot\left(D u_{1}-D u_{2}\right)\right| \\
& \leq \int_{C(h, k)}\left|a\left(x, D u_{1}\right) \cdot D u_{1}\right|+\int_{C(h, k)}\left|\mathbf{a}\left(x, D u_{1}\right) \cdot D u_{2}\right| .
\end{aligned}
$$

Then, by Hölder's inequality, we deduce

$$
\begin{aligned}
& \left|L_{h, k}^{2}\right| \\
& \leq\left(\int_{C(h, k)}\left|\mathbf{a}\left(x, D u_{1}\right)\right|^{p^{\prime}}\right)^{1 / p^{\prime}}\left(\left(\int_{C(h, k)}\left|D u_{1}\right|^{p}\right)^{1 / p}+\left(\int_{C(h, k)}\left|D u_{2}\right|^{p}\right)^{1 / p}\right) .
\end{aligned}
$$

Now, by $\left(H_{2}\right)$,

$$
\begin{aligned}
\left(\int_{C(h, k)}\left|\mathbf{a}\left(x, D u_{1}\right)\right|^{p^{\prime}}\right)^{1 / p^{\prime}} & \leq\left(\int_{C(h, k)} \sigma^{p^{\prime}}\left(\theta(x)+\left|D u_{1}\right|^{p-1}\right)^{p^{\prime}}\right)^{1 / p^{\prime}} \\
& \leq \sigma 2^{\frac{1}{p}}\left(\|\theta\|_{p^{\prime}}^{p^{\prime}}+\int_{\left\{h<\left|u_{1}\right|<k+h\right\}}\left|D u_{1}\right|^{p}\right)^{1 / p^{\prime}}
\end{aligned}
$$

Hence by lemma 3.6, we obtain

$$
\lim _{h \rightarrow \infty} L_{h, k}^{2}=0
$$


Similarly, $\lim _{h \rightarrow \infty} L_{h, k}^{1}=0$. Therefore by (4.5), (4.4) holds. Now, by (4.3) and (4.4),

$$
\lim _{h \rightarrow+\infty} \int_{\left\{\left|u_{1}\right|<h,\left|u_{2}\right|<h\right\}}\left(\mathbf{a}\left(x, D u_{1}\right)-\mathbf{a}\left(x, D u_{2}\right)\right) \cdot D T_{k}\left(u_{1}-u_{2}\right)=0 .
$$

Consequently, for any $h>0, D T_{h}\left(u_{1}\right)=D T_{h}\left(u_{2}\right)$ a.e. in $\Omega$. Then, there exists a constant $c$ such that

$$
u_{1}-u_{2}=c \text { a.e. in } \Omega \text {. }
$$

Applying (4.3) and (4.4) again,

$$
\int_{\Omega}\left(z_{1}-z_{2}\right) T_{k}\left(u_{1}-u_{2}\right)+\int_{\partial \Omega}\left(w_{1}-w_{2}\right) T_{k}\left(u_{1}-u_{2}\right)=0 \quad \forall k>0,
$$

which implies that

$$
\left(w_{1}-w_{2}\right) \chi_{\left\{u_{1}-u_{2} \neq 0\right\}}=0 \text { a.e. in } \partial \Omega,
$$

and

$$
\left(z_{1}-z_{2}\right) \chi_{\left\{u_{1}-u_{2} \neq 0\right\}}=0 \text { a.e. in } \Omega .
$$

Then, if $c \neq 0$ it follows that $w_{1}=w_{2}$, and $z_{1}=z_{2}$.

In order to show that $z_{1}=z_{2}$ in the case $c=0$, we take $T_{h}\left(u_{1}\right)-\varphi$ and $T_{h}\left(u_{1}\right)+\varphi, \varphi \in D(\Omega)$, as test functions in (3.1) for the solution $\left[u_{1}, z_{1}, w_{1}\right]$ and $\left[u_{1}, z_{2}, w_{2}\right]$, respectively, adding these inequalities and letting $h$ go to $+\infty$, if $k>$ $\|\varphi\|_{\infty}$, we get

$$
\lim _{h \rightarrow \infty} J_{h, k}+\int_{\Omega}\left(z_{1}-z_{2}\right) \varphi \leq 0,
$$

where

$$
\begin{aligned}
J_{h, k} & =\int_{\Omega} \mathbf{a}\left(x, D u_{1}\right) \cdot\left[D T_{k}\left(u_{1}-T_{h}\left(u_{1}\right)+\varphi\right)+D T_{k}\left(u_{1}-T_{h}\left(u_{1}\right)-\varphi\right)\right] \\
& =\int_{\left\{\left|u_{1}\right|>h\right\}} \mathbf{a}\left(x, D u_{1}\right) \cdot\left[D T_{k}\left(u_{1}-T_{h}\left(u_{1}\right)+\varphi\right)+D T_{k}\left(u_{1}-T_{h}\left(u_{1}\right)-\varphi\right)\right] .
\end{aligned}
$$

Using Hölder's inequality and Lemma 3.6, we obtain that

$$
\lim _{h \rightarrow \infty} J_{h, k}=0 .
$$

Hence

$$
\int_{\Omega} z_{1} \varphi \leq \int_{\Omega} z_{2} \varphi .
$$

Similarly,

$$
\int_{\Omega} z_{2} \varphi \leq \int_{\Omega} z_{1} \varphi .
$$

Therefore $z_{1}=z_{2}$. 
If $c \neq 0$, following the arguments of [8, Lemma 3.5], we have that $z_{1}=z_{2}$ is constant. In fact, since $\gamma=\partial j, j(r)=\int_{0}^{r} \gamma^{0}(s) d s$, and $z_{1}(x) \in \gamma\left(u_{1}(x)\right) \cap$ $\gamma\left(u_{1}(x)+c\right)$ a.e. $x \in \Omega, j\left(u_{1}(x)+c\right)-j\left(u_{1}(x)\right)=c z_{1}(x)$ a.e. in $\Omega$. Moreover, if $\gamma(\mathbb{R})$ is bounded, $j$ is Lipschitz continuous, $j\left(T_{k}\left(u_{1}\right)+c\right), j\left(T_{k}\left(u_{1}\right)\right) \in W^{1, p}(\Omega)$ and $\nabla\left(j\left(T_{k}\left(u_{1}\right)+c\right)-j\left(T_{k}\left(u_{1}\right)\right)\right)=0$ a.e. in $\Omega$. The above identity is obvious if $\left|u_{1}\right| \geq k$. In the case $\left|u_{1}\right|<k$, we have $\nabla\left(j\left(u_{1}+c\right)-j\left(u_{1}\right)\right)=0$. Therefore $j\left(T_{k}\left(u_{1}\right)+c\right)-j\left(T_{k}\left(u_{1}\right)\right)$ is constant (this constant, in fact, does not depend on $k$ ). Consequently $c z_{1}$ is constant, and since $c \neq 0, z_{1}$ is constant. In the case $\gamma$ is not bounded, we work, truncating $\gamma$, as in [8, Lemma 3.].

Finally let us show $w_{1}=w_{2}$. We take as test function in (3.1) $v=T_{h}\left(u_{i}\right) \pm \varphi$, $\varphi \in W^{1, p}(\Omega) \cap L^{\infty}(\Omega)$, for the entropy solution that involves $w_{i}, i=1,2$. Then, since $u_{1}=u_{2}+c$ and $z_{1}=z_{2}$, we get

$$
\int_{\partial \Omega} w_{1} \varphi=\int_{\partial \Omega} w_{2} \varphi
$$

Consequently $w_{1}=w_{2}$.

In order to get the existence of solutions we need first to prove the following lemma which is a key result.

Lemma 4.2. Let $\Omega$ be a bounded domain in $\mathbb{R}^{N}$ with $\partial \Omega$ of class $C^{1}$. Given $\mu \in$ $\mathfrak{M}_{b}^{p}(\bar{\Omega})$, there exists a sequence $\left\{\psi_{n}\right\}_{n \in \mathbb{N}} \subset C_{c}(\Omega)$,

$$
\psi_{n} \rightarrow \mu \text { as measures, }
$$

such that, for any $\left\{v_{n}\right\}_{n \in \mathbb{N}} \in W^{1, p}(\Omega)$ with $v_{n} \rightarrow v$ weakly in $W^{1, p}(\Omega)$ and all $k>0$,

$$
\lim _{n \rightarrow \infty} \int_{\Omega} T_{k}\left(v_{n}\right) \psi_{n}=\int_{\bar{\Omega}} T_{k}(v) d \mu .
$$

Moreover, if $\mu=f+\operatorname{div} F, f \in L^{p^{\prime}}\left(U_{\Omega}\right), F \in L^{p^{\prime}}\left(U_{\Omega}\right)^{N}$, then

$$
\begin{aligned}
\left|\int_{\Omega} v_{n} \psi_{n}\right| \leq & C_{1}\|f\|_{L^{p^{\prime}}\left(U_{\Omega}\right)}\left\|v_{n}\right\|_{L^{p}(\Omega)} \\
& +C_{2}\|F\|_{\left(L^{p^{\prime}}\left(U_{\Omega}\right)\right)^{N}}\left(\left\|v_{n}\right\|_{L^{p}(\Omega)}+\left\|D v_{n}\right\|_{L^{p}(\Omega)}\right) .
\end{aligned}
$$

Proof. Using the notation of the proof of Lemma 2.1, let $\left\{\theta_{i}\right\}_{i=0,1, \ldots, k}$ be a partition of unity subordinate to $\partial \Omega$ and $U_{1}, \ldots U_{k}$, where

$$
\partial \Omega \subset \bigcup_{i=1}^{k} U_{i},
$$

and let $G_{i}: Q \rightarrow U_{i}$ bijective functions such that $G_{i} \in C^{1}(\bar{Q}), G_{i}{ }^{-1} \in C^{1}\left(\overline{U_{i}}\right)$, $G_{i}\left(Q_{+}\right)=U_{i} \cap \Omega$ and $G_{i}\left(Q_{0}\right)=U_{i} \cap \partial \Omega$. 
Let us write

$$
\mu=\sum_{i=0}^{k} \sigma_{i}, \quad \text { where } \sigma_{i}=\theta_{i} \mu
$$

Let $d_{\epsilon}\left(x^{\prime}, x_{N}\right)=\left(x^{\prime},(1-\epsilon) x_{N}+\epsilon\right)$. Writing $d_{n}^{i}=G_{i} \circ d_{\frac{1}{n}} \circ G_{i}^{-1}, i=1, \ldots, n$, we can define the approximation functions of $\mu$ as follows,

$$
\psi_{n}=\left(\sum_{i=1}^{k} d_{n}^{i} \# \sigma_{i}+\sigma_{0}\right) * \rho_{\frac{1}{2 n}}
$$

where $\rho_{\epsilon}$ is a mollifier with support in $\overline{B(0, \epsilon)}$. Obviously $\psi_{n} \in C_{c}(\Omega)$ and it is easy to see that it satisfies (4.7).

Let $\mu=f+\operatorname{div} F, f \in L^{1}\left(U_{\Omega}\right), F \in L^{p^{\prime}}\left(U_{\Omega}\right)^{N}$. Denoting with the same name the extended functions to $W_{0}^{1, p}\left(U_{\Omega}\right)$, for $u \in W^{1, p}(\Omega)$ we have

$$
\begin{aligned}
\int_{\Omega} u \psi_{n} & =\int_{U_{\Omega}} u \psi_{n}=\int_{U_{\Omega}} u\left(\sum_{i=1}^{k} d_{n}^{i} \# \sigma_{i}+\sigma_{0}\right) * \rho_{\frac{1}{2 n}} \\
& =\int_{U_{\Omega}} u * \rho_{\frac{1}{2 n}} d\left(\sum_{i=1}^{k} d_{n}^{i} \# \sigma_{i}+\sigma_{0}\right) \\
& =\sum_{i=1}^{k} \int_{U_{\Omega} \cap U_{i}} u * \rho_{\frac{1}{2 n}} d\left(d_{n}^{i} \# \sigma_{i}\right)+\int_{U_{\Omega} \cap U_{0}} u * \rho_{\frac{1}{2 n}} d \sigma_{0} \\
& =\sum_{i=1}^{k} \int_{U_{\Omega}} \theta_{i}\left(\left(u * \rho_{\frac{1}{2 n}}\right) \circ d_{n}^{i}\right) d \mu+\int_{U_{\Omega}} \theta_{0}\left(u * \rho_{\frac{1}{2 n}}\right) d \mu .
\end{aligned}
$$

Then,

$$
\begin{aligned}
\int_{\Omega} u \psi_{n}= & \int_{U_{\Omega}}\left(\sum_{i=1}^{k} \theta_{i}\left(\left(u * \rho_{\frac{1}{2 n}}\right) \circ d_{n}^{i}\right)+\theta_{0}\left(u * \rho_{\frac{1}{2 n}}\right)\right) d \mu \\
= & \int_{U_{\Omega}} f\left(\sum_{i=1}^{k} \theta_{i}\left(\left(u * \rho_{\frac{1}{2 n}}\right) \circ d_{n}^{i}\right)+\theta_{0}\left(u * \rho_{\frac{1}{2 n}}\right)\right) \\
& -\int_{U_{\Omega}} F \cdot D\left(\sum_{i=1}^{k} \theta_{i}\left(\left(u * \rho_{\frac{1}{2 n}}\right) \circ d_{n}^{i}\right)+\theta_{0}\left(u * \rho_{\frac{1}{2 n}}\right)\right) .
\end{aligned}
$$


Let us now prove (4.8). Setting $u=T_{k}\left(v_{n}\right)$ in (4.11), $k>0$, and writing $\eta_{n}:=$ $T_{k}\left(v_{n}\right) * \rho_{\frac{1}{2 n}}$, we get

$$
\begin{aligned}
\int_{\Omega} T_{k}\left(v_{n}\right) \psi_{n}= & \int_{U_{\Omega}}\left(\sum_{i=1}^{k} \theta_{i}\left(\eta_{n} \circ d_{n}^{i}\right)+\theta_{0} \eta_{n}\right) d \mu \\
= & \int_{U_{\Omega}} f\left(\sum_{i=1}^{k} \theta_{i}\left(\eta_{n} \circ d_{n}^{i}\right)+\theta_{0} \eta_{n}\right) \\
& -\int_{U_{\Omega}} F \cdot D\left(\sum_{i=1}^{k} \theta_{i}\left(\eta_{n} \circ d_{n}^{i}\right)+\theta_{0} \eta_{n}\right) .
\end{aligned}
$$

To pass to the limit in (4.12), we first see that

$$
\tilde{\eta}_{n}:=\sum_{i=1}^{k} \theta_{i}\left(\eta_{n} \circ d_{n}^{i}\right)+\theta_{0} \eta_{n} \rightarrow T_{k}(v) \text { in } L^{1}(\Omega)
$$

Indeed, by Lusin's theorem, given $\epsilon>0$, there exists a continuous function in $\Omega, \eta^{\epsilon}$, with $\left\|\eta^{\epsilon}\right\|_{L^{\infty}(\Omega)} \leq\left\|T_{k}(v)\right\|_{L^{\infty}(\Omega)}$, such that, if $A^{\epsilon}=\left\{x \in \Omega: \eta^{\epsilon}(x) \neq\right.$ $\left.T_{k}(v)(x)\right\},\left|A^{\epsilon}\right|<\epsilon$. Then,

$$
\begin{aligned}
\left\|\tilde{\eta}_{n}-T_{k}(v)\right\|_{L^{1}(\Omega)}= & \int_{\Omega}\left|\sum_{i=1}^{k} \theta_{i}\left(\eta_{n} \circ d_{n}^{i}\right)+\theta_{0} \eta_{n}-T_{k}(v)\right| \\
\leq & \int_{\Omega}\left|\sum_{i=1}^{k} \theta_{i}\left(\eta_{n} \circ d_{n}^{i}\right)+\theta_{0} \eta_{n}-\sum_{i=1}^{k} \theta_{i}\left(T_{k}(v) \circ d_{n}^{i}\right)-\theta_{0} T_{k}(v)\right| \\
& +\int_{\Omega}\left|\sum_{i=1}^{k} \theta_{i}\left(T_{k}(v) \circ d_{n}^{i}\right)+\theta_{0} T_{k}(v)-\sum_{i=1}^{k} \theta_{i}\left(\eta^{\epsilon} \circ d_{n}^{i}\right)-\theta_{0} \eta^{\epsilon}\right| \\
& +\int_{\Omega}\left|\sum_{i=1}^{k} \theta_{i}\left(\eta^{\epsilon} \circ d_{n}^{i}\right)+\theta_{0} \eta^{\epsilon}-\eta^{\epsilon}\right|+\int_{\Omega}\left|\eta^{\epsilon}-T_{k}(v)\right| \\
\leq & C \int_{\Omega}\left|\eta_{n}-T_{k}(v)\right|+C \int_{A^{\epsilon}}\left|T_{k}(v)-\eta^{\epsilon}\right| \\
& +\int_{\Omega}\left|\sum_{i=1}^{k} \theta_{i}\left(\eta^{\epsilon} \circ d_{n}^{i}\right)+\theta_{0} \eta^{\epsilon}-\eta^{\epsilon}\right|+\int_{A^{\epsilon}}\left|\eta^{\epsilon}-T_{k}(v)\right|,
\end{aligned}
$$


where in the last inequality we have used that the Jacobian $\left|J\left(\left(d_{n}^{i}\right)^{-1}\right)\right|$ is uniformly bounded. Since $\eta^{\epsilon}$ is continuous, $\eta^{\epsilon} \circ d_{n}^{i} \rightarrow \eta^{\epsilon}$ uniformly. Hence,

$$
\lim _{n \rightarrow \infty} \int_{\Omega}\left|\sum_{i=1}^{k} \theta_{i}\left(\eta^{\epsilon} \circ d_{n}^{i}\right)+\theta_{0} \eta^{\epsilon}-\eta^{\epsilon}\right|=0 .
$$

Then, for $\epsilon>0$ fixed, taking limits as $n$ goes to $+\infty$, we get

$$
\limsup _{n}\left\|\tilde{\eta}_{n}-T_{k}(v)\right\|_{L^{1}(\Omega)} \leq 2(C+1)\left\|T_{k}(v)\right\|_{L^{\infty}(\Omega)} \epsilon .
$$

Therefore (4.13) follows, and the first term of the right side of (4.12) converges, as $n \rightarrow \infty$, to $\int_{U_{\Omega}} f T_{k}(v)$.

For the remaining term, we use again (4.13) with the fact that, since the Jacobian $\left|J\left(d_{n}^{i}\right)\right|$ is uniformly bounded,

$$
\left\|\tilde{\eta}_{n}\right\|_{W_{0}^{1, p}\left(U_{\Omega}\right)} \leq C_{1}\left\|v_{n}\right\|_{W^{1, p}(\Omega)} .
$$

We deduce, by taking a subsequence if necessary, that

$$
\tilde{\eta}_{n} \rightarrow T_{k}(v) \text { weakly in } W_{0}^{1, p}\left(U_{\Omega}\right) \text { and a.e. in } U_{\Omega} \text {. }
$$

So, since the limit is independent of the subsequence, the second term of the right side of (4.12) converges, as $n \rightarrow \infty$, to $\int_{U_{\Omega}} F \cdot D T_{k}(v)$. Therefore, passing to the limit in (4.12), we get

$$
\lim _{n \rightarrow \infty} \int_{\Omega} T_{k}\left(v_{n}\right) \psi_{n}=\int_{U_{\Omega}} f T_{k}(v) d x-\int_{U_{\Omega}} F \cdot D T_{k}(v)=\int_{\bar{\Omega}} T_{k}(v) d \mu,
$$

which finishes the proof of (4.8).

Finally, let us prove (4.9). Setting $u=v_{n}$ in (4.11),

$$
\begin{aligned}
\int_{\Omega} v_{n} \psi_{n}= & \int_{U_{\Omega}} f\left(\sum_{i=1}^{k} \theta_{i}\left(\left(v_{n} * \rho_{\frac{1}{2 n}}\right) \circ d_{n}^{i}\right)+\theta_{0}\left(v_{n} * \rho_{\frac{1}{2 n}}\right)\right) \\
& +\int_{U_{\Omega}} F \cdot D\left(\sum_{i=1}^{k} \theta_{i}\left(\left(v_{n} * \rho_{\frac{1}{2 n}}\right) \circ d_{n}^{i}\right)+\theta_{0}\left(v_{n} * \rho_{\frac{1}{2 n}}\right)\right) .
\end{aligned}
$$

Again, it is easy to see that

$$
\begin{aligned}
& \left\|\left(\sum_{i=1}^{k} \theta_{i}\left(\left(v_{n} * \rho_{\frac{1}{2 n}}\right) \circ d_{n}^{i}\right)+\theta_{0}\left(v_{n} * \rho_{\frac{1}{2 n}}\right)\right)\right\|_{W_{0}^{1, p}\left(U_{\Omega}\right)} \\
& \leq C_{2}\left(\left\|v_{n}\right\|_{L^{p}(\Omega)}+\left\|D v_{n}\right\|_{L^{p}(\Omega)}\right) .
\end{aligned}
$$

Now, since $f \in L^{p^{\prime}}\left(U_{\Omega}\right)$ and $F \in L^{p^{\prime}}\left(U_{\Omega}\right)^{N}$, applying Hölder's inequality, (4.9) follows from (4.14). 
Remark 4.3. Observe that if $\Omega$ is an star-shaped domain, that is,

"there exists $x_{0} \in \Omega$ such that $x-\epsilon\left(x-x_{0}\right) \in \Omega, \forall x \in \Omega, \forall \epsilon \in[0,1]$ ", we can simplify the above construction. In fact, setting

$$
d_{\epsilon}(x):=x-\epsilon\left(x-x_{0}\right),
$$

we have that, for $n \geq 2$,

$$
\psi_{n}=\left(d_{\frac{1}{n}} \# \mu\right) * \rho_{\frac{1}{2 n}} \in C_{c}(\Omega)
$$

satisfies (4.7), (4.13) is true for $\tilde{\eta}_{n}=\eta_{n} \circ d_{\frac{1}{n}}$, and (4.8) and (4.9) hold.

We use the following lemma proved in [3, Lemma 4.2].

Lemma 4.4. Let $\left\{u_{n}\right\}_{n \in \mathbb{N}} \subset W^{1, p}(\Omega),\left\{z_{n}\right\}_{n \in \mathbb{N}} \subset L^{1}(\Omega),\left\{w_{n}\right\}_{n \in \mathbb{N}} \subset L^{1}(\partial \Omega)$ such that, for every $n \in \mathbb{N}, z_{n} \in \gamma\left(u_{n}\right)$ a.e. in $\Omega$ and $w_{n} \in \beta\left(u_{n}\right)$ a.e. in $\partial \Omega$. Let us suppose that

(i) if $\mathcal{R}_{\gamma, \beta}^{+}=+\infty$, there exists $M>0$ such that

$$
\int_{\Omega} z_{n}^{+} d x+\int_{\partial \Omega} w_{n}^{+} d \sigma<M \quad \forall n \in \mathbb{N}
$$

(ii) if $\mathcal{R}_{\gamma, \beta}^{+}<+\infty$, there exists $M \in \mathbb{R}$ such that

$$
\int_{\Omega} z_{n} d x+\int_{\partial \Omega} w_{n} d \sigma<M<\mathcal{R}_{\gamma, \beta}^{+}
$$

and

$$
\lim _{L \rightarrow+\infty}\left(\int_{\left\{x \in \Omega: z_{n}(x)<-L\right\}}\left|z_{n}\right| d x+\int_{\left\{x \in \partial \Omega: w_{n}(x)<-L\right\}}\left|w_{n}\right| d \sigma\right)=0
$$

uniformly in $n \in \mathbb{N}$.

Then, there exists a constant $C=C(M)$ such that

$$
\left\|u_{n}^{+}\right\|_{L^{p}(\Omega)} \leq C\left(\left\|D u_{n}^{+}\right\|_{L^{p}(\Omega)}+1\right) \quad \forall n \in \mathbb{N} .
$$

The next theorem gives the existence results of weak and entropy solutions. A contraction principle for weak subsolutions and supersolutions, whose definitions are the standard ones, is also stated.

Theorem 4.5. Let $\Omega \subset \mathbb{R}^{N}$ be an open bounded set with boundary $\partial \Omega$ of class $C^{1}$. Assume $\operatorname{Dom}(\gamma)=\operatorname{Dom}(\beta)=\mathbb{R}$ and $J(\gamma)$ and $J(\beta)$ are bounded (see (2.3) for the definiton of $J$ ). Then: 
(i) For any measures $\mu_{1}, \mu_{2}$ such that $\mu_{1}=\mu_{1}\left\llcorner\Omega, \mu_{2}=\mu_{2}\left\llcorner\partial \Omega, \mu=\mu_{1}+\right.\right.$ $\mu_{2}=f+\operatorname{div}(F), f \in L^{p^{\prime}}\left(U_{\Omega}\right), F \in\left(L^{p^{\prime}}\left(U_{\Omega}\right)\right)^{N}$, and $\mu(\bar{\Omega}) \in \mathcal{R}_{\gamma, \beta}$, there exists a weak solution $[u, z, w]$ of problem $\left(S_{\mu_{1}, \mu_{2}}^{\gamma, \beta}\right)$.

(ii) If $[u, z, w]$ is a weak subsolution of problem $\left(S_{\mu_{1}, \mu_{2}}^{\gamma, \beta}\right), \mu_{1}, \mu_{2}$ measures, $\mu_{1}=$ $\mu_{1}\left\llcorner\Omega\right.$ and $\mu_{2}=\mu_{2}\left\llcorner\partial \Omega\right.$ such that $\mu=\mu_{1}+\mu_{2} \in \mathfrak{M}_{b}^{p}(\bar{\Omega})$, and $[\tilde{u}, \tilde{z}, \tilde{w}]$ is a weak supersolution of problem $\left(S_{\tilde{\mu}_{1}, \tilde{\mu}_{2}}^{\gamma, \beta}\right), \tilde{\mu}_{1}, \tilde{\mu}_{2}$ measures, $\tilde{\mu}_{1}=\tilde{\mu}_{1}\llcorner\Omega$ and $\tilde{\mu}_{2}=\tilde{\mu}_{2}\left\llcorner\partial \Omega\right.$ such that $\tilde{\mu}=\tilde{\mu}_{1}+\tilde{\mu}_{2} \in \mathfrak{M}_{b}^{p}(\bar{\Omega})$, then

$$
\int_{\Omega}(z-\tilde{z})^{+}+\int_{\partial \Omega}(w-\tilde{w})^{+} \leq(\mu-\tilde{\mu})^{+}(\bar{\Omega}) .
$$

(iii) For any measures $\mu_{1}, \mu_{2}$ such that $\mu_{1}=\mu_{1}\left\llcorner\Omega, \mu_{2}=\mu_{2}\left\llcorner\partial \Omega, \mu=\mu_{1}+\right.\right.$ $\mu_{2} \in \mathfrak{M}_{b}^{p}(\bar{\Omega})$, and $\mu(\bar{\Omega}) \in \mathcal{R}_{\gamma, \beta}$, there exists an entropy solution $[u, z, w]$ of problem $\left(S_{\mu_{1}, \mu_{2}}^{\gamma, \beta}\right)$.

Proof. (i): We divide the proof of existence in several steps.

Step 1 . We first suppose that $\mathcal{R}_{\gamma, \beta}=\mathbb{R}$.

Under the assumptions on $\gamma$ and $\beta$, there exist $a_{\gamma}, b_{\gamma},-\infty<a_{\gamma}<0<b_{\gamma}<+\infty$, and $a_{\beta}, b_{\beta},-\infty<a_{\beta}<0<b_{\beta}<+\infty$, such that

$$
a_{\gamma}<\inf \{J(\gamma)\} \leq \sup \{J(\gamma)\}<b_{\gamma}
$$

and

$$
a_{\beta}<\inf \{J(\beta)\} \leq \sup \{J(\beta)\}<b_{\beta} .
$$

We decompose $\gamma$ as follows, $\gamma=\gamma^{d}+\gamma^{c}$, where $\gamma^{d}$ and $\gamma^{c}$ are the following maximal monotone graphs.

$$
\gamma^{d}(r)=\left\{\begin{array}{l}
\gamma(r), \text { if } r \in] a_{\gamma}, b_{\gamma}[, \\
\gamma\left(a_{\gamma}\right), \text { if } r \leq a_{\gamma}, \\
\gamma\left(b_{\gamma}\right), \text { if } r \geq b_{\gamma},
\end{array}\right.
$$

and

$$
\gamma^{c}(r)= \begin{cases}0, & \text { if } r \in] a_{\gamma}, b_{\gamma}[, \\ \gamma(r)-\gamma\left(a_{\gamma}\right), & \text { if } r \leq a_{\gamma}, \\ \gamma(r)-\gamma\left(b_{\gamma}\right), & \text { if } r \geq b_{\gamma} .\end{cases}
$$

In a similar way we decompose $\beta=\beta^{d}+\beta^{c}$.

We now consider a sequence of approximated problems to which we apply Theorem 2.2. Let $\psi_{n}$ given by (4.10). Since $\psi_{n} \in L^{\infty}(\Omega)$, by Theorem 2.2, the problem $\left(S_{\psi_{n}, 0}^{\gamma, \beta}\right)$ has a weak solution $\left[u_{n}, z_{n}, w_{n}\right] \in W^{1, p}(\Omega) \times V^{1, p}(\Omega) \times$ 
$V^{1, p}(\partial \Omega)$, that is, $z_{n}=z_{n}^{d}+z_{n}^{c}, z_{n}^{d}(x) \in \gamma^{d}\left(u_{n}(x)\right)$ a.e. in $\Omega, z_{n}^{c}(x)=\gamma^{c}\left(u_{n}(x)\right)$ a.e. in $\Omega, w_{n}=w_{n}^{d}+w_{n}^{c}, w_{n}^{d}(x) \in \beta^{d}\left(u_{n}(x)\right)$ a.e. in $\partial \Omega, w_{n}^{c}(x)=\beta^{c}\left(u_{n}(x)\right)$ a.e. in $\partial \Omega$, and

$$
\int_{\Omega} \mathbf{a}\left(x, D u_{n}\right) \cdot D v+\int_{\Omega} z_{n} v+\int_{\partial \Omega} w_{n} v=\int_{\Omega} \psi_{n} v \quad \forall v \in W^{1, p}(\Omega) .
$$

Moreover, for any $n$, we have

$$
\begin{gathered}
\left\|z_{n}^{d}\right\|_{\infty} \leq \sup \left\{\left|\gamma\left(a_{\gamma}\right)\right|,\left|\gamma\left(b_{\gamma}\right)\right|\right\},\left\|w_{n}^{d}\right\|_{\infty} \leq \sup \left\{\left|\beta\left(a_{\beta}\right)\right|,\left|\beta\left(b_{\beta}\right)\right|\right\} \\
\left\|w_{n}^{ \pm}\right\|_{L^{1}(\partial \Omega)}+\left\|z_{n}^{ \pm}\right\|_{L^{1}(\Omega)} \leq\left\|\psi_{n}^{ \pm}\right\|_{L^{1}(\Omega)}, \\
\int_{\Omega} z_{n}+\int_{\partial \Omega} w_{n}=\int_{\Omega} \psi_{n} .
\end{gathered}
$$

Taking $v=u_{n}$ in (4.16) and having in mind $\left(H_{1}\right)$, we get

$$
\Lambda \int_{\Omega}\left|D u_{n}\right|^{p} \leq \int_{\Omega} \mathbf{a}\left(x, D u_{n}\right) \cdot D u_{n}+\int_{\Omega} z_{n} u_{n}+\int_{\partial \Omega} w_{n} u_{n}=\int_{\Omega} \psi_{n} u_{n} .
$$

By (4.19) and (4.9), we obtain that

$$
\begin{aligned}
\Lambda \int_{\Omega}\left|D u_{n}\right|^{p} \leq & C_{1}\|f\|_{L^{p^{\prime}}\left(U_{\Omega}\right)}\left\|u_{n}\right\|_{L^{p}(\Omega)} \\
& +C_{2}\|F\|_{\left(L^{p^{\prime}}\left(U_{\Omega}\right)\right)^{N}}\left(\left\|u_{n}\right\|_{L^{p}(\Omega)}+\left\|D u_{n}\right\|_{L^{p}(\Omega)}\right) .
\end{aligned}
$$

By (4.20), (4.17) and Lemma 4.4, we have $\left\{u_{n}\right\}$ bounded in $W^{1, p}(\Omega)$. Then, we can suppose that there exists $u \in W^{1, p}(\Omega)$ such that

$$
\begin{gathered}
u_{n} \text { converges to } u \text { weakly in } W^{1, p}(\Omega), \\
u_{n} \text { converges to } u \text { in } L^{p}(\Omega) \text { and a.e. in } \Omega
\end{gathered}
$$

and

$$
u_{n} \text { converges to } u \text { in } L^{p}(\partial \Omega) \text { and a.e. in } \partial \Omega \text {. }
$$

Arguing as in Proposition 5.1 in [2], it is not difficult to see that $\left\{D u_{n}\right\}$ is a Cauchy sequence in measure. Then, up to extraction of a subsequence, $D u_{n}$ converges to $D u$ a.e. in $\Omega$. Consequently, we obtain that

$$
\mathbf{a}\left(., D u_{n}\right) \text { converges weakly in } L^{p^{\prime}}(\Omega)^{N} \text { and a.e. in } \Omega \text { to } \mathbf{a}(., D u) \text {. }
$$


Since $\gamma^{d}$ and $\beta^{d}$ are bounded we have that there exist subsequences denoted equal such that

$$
z_{n}^{d} \rightarrow z^{d} \in \gamma^{d}(u) \quad \text { weakly* }, z^{d} \in L^{\infty}(\Omega) .
$$

and

$$
w_{n}^{d} \rightarrow w^{d} \in \beta^{d}(u) \quad \text { weakly* }{ }^{*}, w^{d} \in L^{\infty}(\partial \Omega) .
$$

On the other hand, since $\gamma^{c}$ and $\beta^{c}$ are continuous, by (4.21) and (4.22) we have,

$$
z_{n}^{c} \rightarrow z^{c}=\gamma^{c}(u) \text { a.e. in } \Omega,
$$

and

$$
w_{n}^{c} \rightarrow w^{c}=\beta^{c}(u) \quad \text { a.e. on } \partial \Omega .
$$

Then, by Fatou's lemma and having in mind (4.7) and (4.17),

$$
\int_{\Omega}\left|z^{c}\right|+\int_{\partial \Omega}\left|w^{c}\right| \leq \liminf _{n \rightarrow \infty}\left(\int_{\Omega}\left|z_{n}^{c}\right|+\int_{\partial \Omega}\left|w_{n}^{c}\right|\right) \leq \liminf _{n \rightarrow \infty} \int_{\Omega}\left|\psi_{n}\right| \leq|\mu|(\bar{\Omega}) .
$$

Consequently, $z=z^{d}+z^{c} \in L^{1}(\Omega), z \in \gamma(u)$ a.e. in $\Omega$, and $w=w^{d}+w^{c} \in$ $L^{1}(\partial \Omega), w \in \beta(u)$ a.e. on $\partial \Omega$.

Finally, let us prove that $[u, z, w]$ is a weak solution of $\left(S_{\mu_{1}, \mu_{2}}^{\gamma, \beta}\right)$. Let $\hat{v} \in$ $W^{1, p}(\Omega) \cap L^{\infty}(\Omega)$ and $S \in C^{2}(\mathbb{R}) \cap L^{\infty}(\mathbb{R})$ satisfying

$$
\begin{gathered}
S(0)=0,0 \leq S^{\prime} \leq 1, S^{\prime}(s)=0 \text { for } s \text { large enough, } \\
S(-s)=-S(s), \text { and } S^{\prime \prime}(s) \leq 0 \text { for } s \geq 0 .
\end{gathered}
$$

Taking $S\left(u_{n}-\hat{v}\right)$ as test function in (4.16), we get

$$
\begin{aligned}
\int_{\Omega} \mathbf{a}\left(x, D u_{n}\right) \cdot D S\left(u_{n}-\hat{v}\right) & +\int_{\Omega} z_{n} S\left(u_{n}-\hat{v}\right)+\int_{\partial \Omega} w_{n} S\left(u_{n}-\hat{v}\right) \\
& =\int_{\Omega} \psi_{n} S\left(u_{n}-\hat{v}\right) .
\end{aligned}
$$

We can write the first term of (4.26) as

$$
\int_{\Omega} \mathbf{a}\left(x, D u_{n}\right) \cdot D u_{n} S^{\prime}\left(u_{n}-\hat{v}\right)-\int_{\Omega} \mathbf{a}\left(x, D u_{n}\right) \cdot D w S^{\prime}\left(u_{n}-\hat{v}\right) .
$$

Since $u_{n} \rightarrow u$ and $D u_{n} \rightarrow D u$ a.e., Fatou's lemma yields

$$
\int_{\Omega} \mathbf{a}(x, D u) \cdot D u S^{\prime}(u-\hat{v}) \leq \liminf _{n \rightarrow \infty} \int_{\Omega} \mathbf{a}\left(x, D u_{n}\right) \cdot D u_{n} S^{\prime}\left(u_{n}-\hat{v}\right) .
$$


The second term of (4.27) is estimated as follows. By (4.23)

$$
\mathbf{a}\left(x, D u_{n}\right) \rightarrow \mathbf{a}(x, D u) \quad \text { weakly in } L^{p^{\prime}}(\Omega) .
$$

On the other hand,

$$
\left|D \hat{v} S^{\prime}\left(u_{n}-\hat{v}\right)\right| \leq|D \hat{v}| \in L^{p}(\Omega) .
$$

Then, by the Dominated Convergence Theorem, we have

$$
D \hat{v} S^{\prime}\left(u_{n}-\hat{v}\right) \rightarrow D \hat{v} S^{\prime}(u-\hat{v}) \quad \text { in } L^{p}(\Omega)^{N} .
$$

Hence, by (4.28) and (4.29), it follows that

$$
\lim _{n \rightarrow \infty} \int_{\Omega} \mathbf{a}\left(x, D u_{n}\right) \cdot D \hat{v} S^{\prime}\left(u_{n}-\hat{v}\right)=\int_{\Omega} \mathbf{a}(x, D u) \cdot D \hat{v} S^{\prime}(u-\hat{v}) .
$$

On the other hand, writing the second term of (4.29) as

$$
\int_{\Omega} z_{n} S\left(u_{n}-\hat{v}\right)=\int_{\Omega}\left(z_{n}-\gamma^{0}(\hat{v})\right) S\left(u_{n}-\hat{v}\right)+\int_{\Omega} \gamma^{0}(\hat{v}) S\left(u_{n}-\hat{v}\right),
$$

having in mind (4.21), (4.24) and (4.25), and using Fatou's lemma, we get

$$
\int_{\Omega} z S(u-\hat{v}) \leq \liminf _{n \rightarrow \infty} \int_{\Omega} z_{n} S\left(u_{n}-\hat{v}\right) .
$$

Similarly, we can obtain that

$$
\int_{\partial \Omega} w S(u-\hat{v}) \leq \liminf _{n \rightarrow \infty} \int_{\partial \Omega} w_{n} S\left(u_{n}-\hat{v}\right) .
$$

For the right hand side of (4.26), by (4.34), we have

$$
\lim _{n \rightarrow \infty} \int_{\Omega} S\left(u_{n}-\hat{v}\right) \psi_{n}=\int_{U_{\Omega}} f S(u-\hat{v}) d x-\int_{U_{\Omega}} F \cdot D S(u-\hat{v}) .
$$

From (4.30), (4.32), (4.33) and (4.34), taking limits in (4.26), we obtain

$$
\begin{gathered}
\int_{\Omega} \mathbf{a}(x, D u) \cdot D S(u-\hat{v})+\int_{\Omega} z S(u-\hat{v})+\int_{\partial \Omega} w S(u-\hat{v}) \\
\leq \int_{\Omega} S(u-\hat{v}) d \mu_{1}+\int_{\partial \Omega} S(u-\hat{v}) d \mu_{2} .
\end{gathered}
$$

Applying now the technique used in the proof of [6, Lemma 3.2] we get, for any $k>0$,

$$
\begin{aligned}
& \int_{\Omega} \mathbf{a}(x, D u) \cdot D T_{k}(u-\hat{v})+\int_{\Omega} z T_{k}(u-\hat{v})+\int_{\partial \Omega} w T_{k}(u-\hat{v}) \\
& \leq \int_{\Omega} T_{k}(u-\hat{v}) d \mu_{1}+\int_{\partial \Omega} T_{k}(u-\hat{v}) d \mu_{2} .
\end{aligned}
$$


Let now $v \in W^{1, p}(\Omega) \cap L^{\infty}(\Omega)$. Taking $k \geq\|v\|_{\infty}, \hat{v}=T_{h}(u) \pm v$ in (4.35), and letting $h$ go to $+\infty$, we have

$$
\int_{\Omega} \mathbf{a}(x, D u) \cdot D v+\int_{\Omega} z v+\int_{\partial \Omega} w v=\int_{\Omega} v d \mu_{1}+\int_{\partial \Omega} v d \mu_{2},
$$

which finishes the proof of (i) in the case $\mathcal{R}_{\gamma, \beta}=\mathbb{R}$.

Step 2. Let us now suppose that $\mathcal{R}_{\gamma, \beta}^{-}=-\infty$ and $\mathcal{R}_{\gamma, \beta}^{+}$is finite.

By the previous step, there exists $\left[u_{m}, z_{m}+\frac{1}{m} u_{m}^{+}, w_{m}\right]$ a weak solution of problem $\left(S_{\mu_{1}, \mu_{2}}^{\gamma_{m}, \beta}\right)$, where $\gamma_{m}(r)=\gamma(r)+\frac{1}{m} r^{+}$. Now, by (ii), whose proof is independent of (i) (see below), for $m_{1} \leq m_{2}$ we have that

$$
z_{m_{1}}+\frac{1}{m_{2}} u_{m_{1}}^{+} \leq z_{m_{2}}+\frac{1}{m_{2}} u_{m_{2}}^{+} \text {a.e. in } \Omega
$$

and

$$
w_{m_{1}} \leq w_{m_{2}} \text { a.e. in } \partial \Omega .
$$

Moreover, it is easy to see that

$$
u_{m_{1}} \leq u_{m_{2}} \quad \text { a.e. in } \Omega \text {. }
$$

Therefore,

$$
z_{m_{1}} \leq z_{m_{2}} \quad \text { a.e. in } \Omega \text {. }
$$

On the other hand, similar estimates to (4.17), (4.18) and (4.20) can be obtained as in the Step 1. Consequently, $\left\{z_{m}\right\}$ is convergent in $L^{1}(\Omega)$ and $\left\{w_{m}\right\}$ is convergent in $L^{1}(\partial \Omega)$. By Lemma 4.4, we can get that $\left\{u_{n}\right\}$ is bounded in $W^{1, p}(\Omega)$. The proof can be finished as above. step.

The case $\mathcal{R}_{\gamma, \beta}^{+}=+\infty$ and $\mathcal{R}_{\gamma, \beta}^{-}$finite is similar. Let us now prove the last

Step 3. We suppose now that $\mathcal{R}_{\gamma, \beta}^{-}$and $\mathcal{R}_{\gamma, \beta}^{+}$are finite.

By the previous step, let us construct $\left[u_{m}, z_{m}-\frac{1}{m} u_{m}^{-}, w_{m}\right]$ a weak solution of problem $\left(S_{\mu_{1}, \mu_{2}}^{\gamma_{m}, \beta}\right)$, where $\gamma_{m}(r)=\gamma(r)-\frac{1}{m} r^{-}$. Now, by (ii), arguing as in the previous step, we have that, for $m_{1} \leq m_{2}$,

$$
z_{m_{1}} \geq z_{m_{2}} \quad \text { a.e. in } \Omega
$$

and

$$
w_{m_{1}} \geq w_{m_{2}} \text { a.e. in } \partial \Omega .
$$

By Lemma 4.4, we first obtain that $\left\{u_{n}^{-}\right\}$is bounded in $W^{1, p}(\Omega)$ and afterward, on account of this fact, we obtain that $\left\{u_{n}^{+}\right\}$is bounded in $W^{1, p}(\Omega)$. Again, the proof can be finished as in the first step. 
(ii): Let $[u, z, w]$ be a weak subsolution of problem $\left(S_{\mu_{1}, \mu_{2}}^{\gamma, \beta}\right)$ and let $[\tilde{u}, \tilde{z}, \tilde{w}]$ be a weak supersolution of problem $\left(S_{\tilde{\mu}_{1}, \tilde{\mu}_{2}}^{\gamma, \beta}\right)$. Then,

$$
\begin{array}{r}
\int_{\Omega} \mathbf{a}(x, D u) \cdot D v+\int_{\Omega} z v+\int_{\partial \Omega} w v \leq \int_{\bar{\Omega}} v d \mu \\
\forall v \in W^{1, p}(\Omega) \cap L^{\infty}(\Omega), \quad v \geq 0
\end{array}
$$

and

$$
\begin{aligned}
& \int_{\Omega} \mathbf{a}(x, D \tilde{u}) \cdot D v+\int_{\Omega} \tilde{z} v+\int_{\partial \Omega} \tilde{w} v \geq \int_{\bar{\Omega}} v d \tilde{\mu} \\
& \forall v \in W^{1, p}(\Omega) \cap L^{\infty}(\Omega), v \geq 0 .
\end{aligned}
$$

Consider $\rho \in W^{1, p}(\Omega), 0 \leq \rho \leq 1$. Taking as test function $v=\frac{T_{k}^{+}}{k}(u-\tilde{u}+k \rho)$ in (4.36) and in (4.37), we have

$$
\begin{gathered}
\int_{\Omega} \mathbf{a}(x, D u) \cdot D\left(\frac{T_{k}^{+}}{k}(u-\tilde{u}+k \rho)\right)+\int_{\Omega} z \frac{T_{k}^{+}}{k}(u-\tilde{u}+k \rho) \\
\quad+\int_{\partial \Omega} w \frac{T_{k}^{+}}{k}(u-\tilde{u}+k \rho) \leq \int_{\bar{\Omega}}\left(\frac{T_{k}^{+}}{k}(u-\tilde{u}+k \rho)\right) d \mu
\end{gathered}
$$

and

$$
\begin{gathered}
\int_{\Omega} \mathbf{a}(x, D \tilde{u}) \cdot D\left(\frac{T_{k}^{+}}{k}(u-\tilde{u}+k \rho)\right)+\int_{\Omega} \tilde{z} \frac{T_{k}^{+}}{k}(u-\tilde{u}+k \rho) \\
\quad+\int_{\partial \Omega} \tilde{w} \frac{T_{k}^{+}}{k}(u-\tilde{u}+k \rho) \geq \int_{\bar{\Omega}}\left(\frac{T_{k}^{+}}{k}(u-\tilde{u}+k \rho)\right) d \tilde{\mu}
\end{gathered}
$$

Therefore, having in mind the monotonicity of $\mathbf{a}$, we get

$$
\begin{aligned}
& \int_{\Omega}(z-\tilde{z}) \frac{T_{k}^{+}}{k}(u-\tilde{u}+k \rho)+\int_{\partial \Omega}(w-\tilde{w}) \frac{T_{k}^{+}}{k}(u-\tilde{u}+k \rho) \\
& \quad+\int_{\{0<u-\tilde{u}+k \rho<k\}}(\mathbf{a}(x, D u)-\mathbf{a}(x, D \tilde{u})) \cdot D \rho \\
& \leq \int_{\bar{\Omega}}\left(\frac{T_{k}^{+}}{k}(u-\tilde{u}+k \rho)\right) d(\mu-\tilde{\mu}) \leq(\mu-\tilde{\mu})^{+}(\bar{\Omega}) .
\end{aligned}
$$


Taking the limit as $k$ goes to 0 in the above expression, having in mind that $D u=$ $D \tilde{u}$ where $u=\tilde{u}$, we obtain that

$$
\begin{gathered}
\int_{\Omega}(z-\tilde{z}) \operatorname{sign}_{0}^{+}(u-\tilde{u}) \chi_{\{u \neq \tilde{u}\}}+\int_{\Omega}(z-\tilde{z}) \rho \chi_{\{u=\tilde{u}\}} \\
\int_{\partial \Omega}(w-\tilde{w}) \operatorname{sign}_{0}^{+}(u-\tilde{u}) \chi_{\{u \neq \tilde{u}\}}+\int_{\partial \Omega}(w-\tilde{w}) \rho \chi_{\{u=\tilde{u}\}} \\
\leq(\mu-\tilde{\mu})^{+}(\bar{\Omega}) .
\end{gathered}
$$

By approximation we can suppose that (4.38) holds for every $0 \leq \rho \in W^{1,1}(\Omega) \cap$ $L^{\infty}(\Omega)$. It is easy to see that there exist $0 \leq \rho_{n} \in W^{1,1}(\Omega) \cap L^{\infty}(\Omega)$ such that $\rho_{n}=\operatorname{sign}_{0}^{+}(w-\tilde{w}), \mathcal{H}^{N-1}$ a.e. on $\partial \Omega$, and

$$
\rho_{n} \rightarrow \operatorname{sign}_{0}^{+}(z-\tilde{z}) \quad \text { in } L^{1}(\Omega) .
$$

Then, taking $\rho=\rho_{n}$ in (4.38) and sending $n \rightarrow+\infty$, we get the contraction principle (4.15).

(iii): We consider the same steps as in the proof of (i) and only give some details of the proof.

Step 1. In the case $\mathcal{R}_{\gamma, \beta}=\mathbb{R}$, we use the same approximated problem $\left(S_{\psi_{n}, 0}^{\gamma, \beta}\right)$ as above. Then, there exists $\left[u_{n}, z_{n}, w_{n}\right]$ weak solution of $\left(S_{\psi_{n}, 0}^{\gamma, \beta}\right)$ such that

$$
\left\|D T_{k}\left(u_{n}\right)\right\|_{L^{p}(\Omega)}^{p} \leq \frac{k}{\Lambda}\left\|\psi_{n}^{ \pm}\right\|_{L^{1}(\Omega)} .
$$

By (4.39) and (4.7), we have that $\left\{T_{k}\left(u_{n}\right)\right\}$ is bounded in $W^{1, p}(\Omega)$. Therefore, we can suppose that there exists $\sigma_{k} \in W^{1, p}(\Omega)$ such that

$$
\begin{gathered}
T_{k}\left(u_{n}\right) \text { converges to } \sigma_{k} \text { weakly in } W^{1, p}(\Omega), \\
T_{k}\left(u_{n}\right) \text { converges to } \sigma_{k} \text { in } L^{p}(\Omega) \text { and a.e. in } \Omega
\end{gathered}
$$

and

$$
T_{k}\left(u_{n}\right) \text { converges to } \sigma_{k} \text { in } L^{p}(\partial \Omega) \text { and a.e. in } \partial \Omega \text {. }
$$

Let us show that $u_{n}$ converges almost every where in $\Omega$. By (4.7), there exists $M>0$ such that,

$$
\int_{\Omega} z_{n}^{+}+\int_{\partial \Omega} w_{n}^{+}<M \quad \forall n \in \mathbb{N} .
$$

In particular, if

$$
z_{n}^{k}= \begin{cases}z_{n} & \text { if }\left|u_{n}\right|<k, \\ \gamma^{0}(k) & \text { if } u_{n} \geq k, \\ \gamma^{0}(-k) & \text { if } u_{n} \leq-k\end{cases}
$$


and

$$
w_{n}^{k}= \begin{cases}w_{n} & \text { if }\left|u_{n}\right|<k, \\ \beta^{0}(k) & \text { if } u_{n} \geq k, \\ \beta^{0}(-k) & \text { if } u_{n} \leq-k,\end{cases}
$$

$k \in \mathbb{N}$, then

$$
\int_{\Omega}\left(z_{n}^{k}\right)^{+}+\int_{\partial \Omega}\left(w_{n}^{k}\right)^{+}<M \quad \forall n, k \in \mathbb{N} .
$$

Since $z_{n}^{k} \in \gamma\left(T_{k}\left(u_{n}\right)\right)$ a.e. in $\Omega$ and $w_{n}^{k} \in \beta\left(T_{k}\left(u_{n}\right)\right)$ a.e. in $\partial \Omega$, by Lemma 4.4, (4.39) and (4.7), we get

$$
\begin{aligned}
\mathcal{L}^{N}\left(\left\{x \in \Omega: \sigma_{k}^{+}(x)=k\right\}\right) & =\int_{\left\{x \in \Omega: \sigma_{k}^{+}(x)=k\right\}} \frac{\left|\sigma_{k}^{+}(x)\right|^{p}}{k^{p}} \\
& \leq \frac{1}{k^{p}} \liminf _{n} \int_{\Omega}\left|T_{k}\left(\left(u_{n}\right)^{+}\right)\right|^{p} \leq \frac{C}{k^{p-1}} .
\end{aligned}
$$

Hence

$$
\mathcal{L}^{N}\left(\left\{x \in \Omega: \sigma_{k}^{+}(x)=k\right\}\right) \rightarrow 0 \quad \text { as } k \rightarrow+\infty .
$$

Working similarly for the negative part $\sigma_{k}^{-}$we obtain

$$
\mathcal{L}^{N}\left(\left\{x \in \Omega: \sigma_{k}^{-}(x)=k\right\}\right) \rightarrow 0 \quad \text { as } k \rightarrow+\infty .
$$

Therefore, if we define $u(x)=\sigma_{k}(x)$ on $\left\{x \in \Omega:\left|\sigma_{k}(x)\right|<k\right\}$, then

$$
u_{n} \text { converges to } u \text { a.e. in } \Omega \text {. }
$$

Consequently we obtain that $\sigma_{k}=T_{k}(u)$ and $u \in \mathcal{T}^{1, p}(\Omega)$.

Let us now see that $u \in \mathcal{T}_{\text {tr }}^{1, p}(\Omega)$. On the one hand we have that $u_{n} \rightarrow u$ a.e. in $\Omega$. On the other hand, since $D T_{k}\left(u_{n}\right)$ is bounded in $L^{p}(\Omega)$ and, as in Proposition 5.1 in [2], $D T_{k}\left(u_{n}\right) \rightarrow D T_{k}(u)$ in measure, it follows from [6, Lemma 6.1] that $D T_{k}\left(u_{n}\right) \rightarrow D T_{k}(u)$ in $L^{1}(\Omega)$. Now, using again Lemma 4.4 , it can be shown that

$$
\mathcal{H}^{N-1}\left(\left\{x \in \partial \Omega:\left|\left(T_{k}(u)\right)(x)\right|=k\right\}\right) \rightarrow 0 \quad \text { as } k \rightarrow+\infty \text {. }
$$

Therefore, if we define $v(x)=T_{k}(u)(x)$ on $\left\{x \in \partial \Omega:\left|T_{k}(u)(x)\right|<k\right\}$, we have that

$$
u_{n} \text { converges to } v \text { a.e. in } \partial \Omega \text {. }
$$

Consequently, $u \in \mathcal{T}_{\text {tr }}^{1, p}(\Omega)$.

These facts are the main ingredients to finish the proof as in Step 2 of (i).

Step 2. We now suppose that $\mathcal{R}_{\gamma, \beta}^{-}=-\infty$ and $\mathcal{R}_{\gamma, \beta}^{+}$is finite and consider again $\gamma_{m}(r)=\gamma(r)+\frac{1}{m} r^{+}$. Then, for every $m$, we obtain an entropy solution $\left[u_{m}, z_{m}, w_{m}\right]$ 
of problem $\left(S_{\mu_{1}, \mu_{2}}^{\gamma_{m}, \beta}\right)$ as limit of weak solutions. By the contraction principle for weak solutions, $\left\{z_{m}\right\}$ and $\left\{w_{m}\right\}$ are non decreasing in $m$. These facts are the main ingredients to finish the proof.

Finally, the third step follows the same idea as the above one.

Remark 4.6. Observe we have the following monotonicity result for entropy solutions.

Let $[u, z, w]$ be an entropy solution of problem $\left(S_{\mu_{1}, \mu_{2}}^{\gamma, \beta}\right), \mu_{1}, \mu_{2}$ measures, $\mu_{1}=$ $\mu_{1}\left\llcorner\Omega\right.$ and $\mu_{2}=\mu_{2}\left\llcorner\partial \Omega\right.$ such that $\mu=\mu_{1}+\mu_{2} \in \mathfrak{M}_{b}^{p}(\bar{\Omega})$ and $\mu(\bar{\Omega}) \in \mathcal{R}_{\gamma, \beta}$, and $[\tilde{u}, \tilde{z}, \tilde{w}]$ an entropy solution of problem $\left(S_{\tilde{\mu}_{1}, \tilde{\mu}_{2}}^{\gamma, \beta}\right), \tilde{\mu}_{1}, \tilde{\mu}_{2}$ measures, $\tilde{\mu}_{1}=\tilde{\mu}_{1}\llcorner\Omega$ and $\tilde{\mu}_{2}=\tilde{\mu}_{2}\left\llcorner\partial \Omega\right.$ such that $\tilde{\mu}=\tilde{\mu}_{1}+\tilde{\mu}_{2} \in \mathfrak{M}_{b}^{p}(\bar{\Omega})$ and $\tilde{\mu}(\bar{\Omega}) \in \mathcal{R}_{\gamma, \beta}$. If $\mu_{1} \leq \mu_{2}$, then $z_{1} \leq z_{2}$ a.e. in $\Omega$ and $w_{1} \leq w_{2}$ a.e. in $\partial \Omega$.

Remark 4.7. We point out that the cases of the Hele-Shaw problem, which corresponds to

$$
\gamma(r)= \begin{cases}0 & \text { if } r<0 \\ {[0,1]} & \text { if } r=0 \\ 1 & \text { if } r>0\end{cases}
$$

and the multiphase Stefan problem, which corresponds to

$$
\gamma(r)= \begin{cases}r-1 & \text { if } r<0 \\ {[-1,0]} & \text { if } r=0 \\ r & \text { if } r>0\end{cases}
$$

are included in the above existence and uniqueness results.

Let us also remark that, as a consequence of the previous results, we have existence and uniqueness of entropy solutions for problem $\left(L_{0, \mu}^{g_{1}, \beta}\right)$ for a large class of maximal monotone graphs $\beta$.

\section{Existence for Dirichlet boundary condition}

In this section we study the particular case of Dirichlet boundary condition, that is, for $\beta$ the monotone graph $D=\{0\} \times \mathbb{R}$, which corresponds to the problem

$$
\left(S_{\mu, 0}^{\gamma, D}\right) \begin{cases}-\operatorname{div} \mathbf{a}(x, D u)+\gamma(u) \ni \mu & \text { in } \Omega \\ u=0 & \text { on } \partial \Omega,\end{cases}
$$

where $\gamma$ is a maximal monotone graph in $\mathbb{R}^{2}$ and $\mu$ a diffuse measure in $\Omega$. In this case the concept of entropy solution is the following: 
Definition 5.1. Let $\mu \in \mathcal{M}_{b}^{p}(\Omega)$. A pair of functions $[u, z] \in \mathcal{T}_{0}^{1, p}(\Omega) \times L^{1}(\Omega)$ is an entropy solution of problem $\left(S_{\mu, 0}^{\gamma, D}\right)$ if $z(x) \in \gamma(u(x))$ a.e. in $\Omega$ and

$$
\int_{\Omega} \mathbf{a}(x, D u) \cdot D T_{k}(u-v) d x+\int_{\Omega} z T_{k}(u-v) d x \leq \int_{\Omega} T_{k}(u-v) d \mu
$$

for all $k>0$ and for all $v \in W_{0}^{1, p}(\Omega) \cap L^{\infty}(\Omega)$.

We have the following existence and uniqueness result.

Theorem 5.2. Let $\Omega \subset \mathbb{R}^{N}$ be an open bounded set with boundary $\partial \Omega$ of class $C^{1}$. Assume $D(\gamma)=\mathbb{R}$ and $J(\gamma)$ is bounded. Then, for any diffuse measure $\mu \in \mathcal{M}_{b}^{p}(\Omega)$, there exists an entropy solution $[u, z]$ of problem $\left(S_{\mu, 0}^{\gamma, D}\right)$.

Moreover, if $[u, z]$ is an entropy solution of problem $\left(S_{\mu, 0}^{\gamma, D}\right), \mu \in \mathcal{M}_{b}^{p}(\Omega)$, and $[\tilde{u}, \tilde{z}]$ is an entropy solution of problem $\left(S_{\tilde{\mu}, 0}^{\gamma, D}\right), \tilde{\mu} \in \mathcal{M}_{b}^{p}(\Omega)$, then

$$
\int_{\Omega}(z-\tilde{z})^{+} \leq(\mu-\tilde{\mu})^{+}(\Omega)
$$

Proof. Let us write $\mu=f+\operatorname{div} F, f \in L^{1}(\Omega), F \in L^{p^{\prime}}(\Omega)^{N}$, and consider $\psi_{n}=T_{n}(f)+\operatorname{div} F$. Working as in the proof of Theorem 4.5 (i) and (ii), on account of Theorem 2.3, problem $\left(S_{\psi_{n}, 0}^{\gamma, D}\right)$ has a unique weak solution $\left[u_{n}, z_{n}\right] \in$ $W_{0}^{1, p}(\Omega) \times L^{1}(\Omega), z_{n} \in \gamma\left(u_{n}\right)$ a.e. in $\Omega$, such that

$$
\int_{\Omega} \mathbf{a}\left(x, D u_{n}\right) \cdot D v+\int_{\Omega} z_{n} v=\int_{\Omega} \psi_{n} v, \quad \forall v \in W_{0}^{1, p}(\Omega) .
$$

Moreover,

$$
\left\|z_{n}-z_{m}\right\|_{L^{1}(\Omega)} \leq\left\|\psi_{n}-\psi_{m}\right\|_{L^{1}(\Omega)} .
$$

Taking $v=T_{k}\left(u_{n}^{ \pm}\right)$in (5.1), and having in mind $\left(H_{1}\right)$, we get

$$
\left\|D T_{k}\left(u_{n}^{ \pm}\right)\right\|_{L^{p}(\Omega)}^{p} \leq \frac{k}{\Lambda}|\mu|(\Omega) .
$$

By (5.2), $\left\{T_{k}\left(u_{n}\right)\right\}$ is bounded in $W_{0}^{1, p}(\Omega)$. Then, we can suppose that there exists $\sigma_{k} \in W_{0}^{1, p}(\Omega)$ such that

$$
T_{k}\left(u_{n}\right) \text { converges to } \sigma_{k} \text { weakly in } W_{0}^{1, p}(\Omega),
$$

and

$$
T_{k}\left(u_{n}\right) \text { converges to } \sigma_{k} \text { in } L^{p}(\Omega) \text { and a.e. in } \Omega \text {. }
$$


Let us prove that $u_{n}$ converges almost everywhere in $\Omega$. By (5.2) and Poincaré's inequality, we have

$$
\begin{aligned}
\mathcal{L}^{N}\left(\left\{x \in \Omega: \sigma_{k}^{+}(x)^{p}=k^{p}\right\}\right) & =\int_{\left\{x \in \Omega: \sigma_{k}^{+}(x)=k\right\}} \frac{\sigma_{k}^{+}(x)^{p}}{k^{p}} \\
& \leq \frac{1}{k^{p}} \liminf _{n} \int_{\Omega}\left|T_{k}\left(\left(u_{n}\right)^{+}\right)\right|^{p} \leq \frac{C}{k^{p-1}} .
\end{aligned}
$$

Working similarly for the negative part and taking into account both results we get

$$
\mathcal{L}^{N}\left(\left\{x \in \Omega:\left|\sigma_{k}(x)\right|=k\right\}\right) \leq \frac{C}{k^{p-1}} .
$$

Therefore, if we define $u(x)=\sigma_{k}(x)$ on $\left\{x \in \Omega:\left|\sigma_{k}(x)\right|<k\right\}$,

$$
u_{n} \text { converges to } u \text { a.e. in } \Omega \text {, }
$$

$$
T_{k}\left(u_{n}\right) \text { converges to } T_{k}(u) \text { weakly in } W_{0}^{1, p}(\Omega) \text {, }
$$

and

$$
T_{k}\left(u_{n}\right) \text { converges to } T_{k}(u) \text { in } L^{p}(\Omega) \text { and a.e. in } \Omega \text {. }
$$

Consequently, we also have that $u \in \mathcal{T}_{0}^{1, p}(\Omega)$ (see [6]). The rest of the proof follows the same ideas to the one given for Theorem 4.5.

For the contraction principle, let us write $\mu=f+\operatorname{div} F, f \in L^{1}(\Omega)$, $F \in L^{p^{\prime}}(\Omega)^{N}, \tilde{\mu}=\tilde{f}+\operatorname{div} \tilde{F}, \tilde{f} \in L^{1}(\Omega), \tilde{F} \in L^{p^{\prime}}(\Omega)^{N}$, and consider approximation measures $\mu_{n}=T_{n}(f)+\operatorname{div} F$ and $\tilde{\mu}_{n}=T_{n}(\tilde{f})+\operatorname{div} \tilde{F}$. As in Theorem 4.5, we can prove a contraction principle for the solutions of the approximated problems corresponding to $\mu_{n}$ and $\tilde{\mu}_{n}$, respectively. Moreover by the uniqueness result proved in Theorem 4.1 we obtain the contraction principle in this case.

\section{References}

[1] F. Andreu, J. M. Mazón, S. Segura de León and J. Toledo, Quasi-linear elliptic and parabolic equations in $L^{1}$ with nonlinear boundary conditions, Adv. Math. Sci. Appl. 7 (1997), 183-213.

[2] F. Andreu, N. Igbida, J. M. Mazón and J. Toledo, $L^{1}$ existence and uniqueness results for quasi-linear elliptic equations with nonlinear boundary conditions, Ann. Inst. H. Poincaré Anal. Non Linéaire 24 (2007), 61-89.

[3] F. Andreu, N. IgBida, J. M. Mazón and J. Toledo, A degenerate elliptic-parabolic problem with nonlinear dynamical boundary conditions, Interfaces Free Bound. 8 (2006), 447-479.

[4] F. Andreu, N. IgBida, J. M. Mazón and J. Toledo, Obstacle problems for degenerate elliptic equations with nonlinear boundary conditions, Math. Models Methods Appl. Sci. 18 (2008), 1869-1893. 
[5] P. BARAS and M. PIERre, Singularités éliminables pour des équations semi-linéaires, Ann. Inst. Fourier (Grenoble) 34 (1984), 185-206.

[6] Ph. Bénilan, L. Boccardo, Th. Gallouët, R. Gariepy, M. Pierre and J. L. VÁZQUEZ, An $L^{1}$-theory of existence and uniqueness of solutions of nonlinear elliptic equations, Ann. Scuola Norm. Sup. Pisa Cl. Sci. (4) 22 (1995), 241-273.

[7] PH. BÉNILAN and H. BREZIS, Nonlinear problems related to the Thomas-Fermi equation, dedicated to Philippe Bénilan, J. Evol. Equ. 3 (2003), 673-770.

[8] Ph. Benilan, H. Brezis and M. G. Crandall, A semilinear equation in $L^{1}\left(R^{N}\right)$, Ann. Scuola Norm. Sup. Pisa Cl. Sci. (4) 2 (1975), 523-555.

[9] Ph. BÉnilan, M. G. CRANDAll and P. SACKS, Some $L^{1}$ existence and dependence results for semilinear elliptic equations under nonlinear boundary conditions, Appl. Math. Optim. 17 (1986), 203-224.

[10] D. Bartolucci, F. Leoni, L. Orsina and A. C. Ponce, Semilinear equations with exponential nonlinearity and measure data, Ann. Inst. H. Poincaré Anal. Non Linéaire 22 (2005), 799-815.

[11] L. BocCARDo and T. GAllouet, Nonlinear elliptic and parabolic equations involving measure data, J. Funct. Anal. 87 (1989), 149-169.

[12] L. BocCARDO and T. GALLOUET, Nonlinear elliptic equations with right-hand side measures, Comm. Partial Differential Equations 17 (1992), 641-655.

[13] L. Boccardo, T. Gallouet and L. Orsina, Existence and uniqueness of entropy solutions for nonlinear elliptic equations with measure data, Ann. Inst. H. Poincaré Anal. Non Linéaire 13 (1996), 539-551.

[14] L. BOCCARDO and F. MURAT, Remarques sur l'homogénéisation de certains problèmes quasi-linéaires. Portugaliae Math. 41 (1982), 535-562.

[15] H. Brezis, Problèmes unilatéraux, J. Math. Pures Appl. 51 (1972), 1-168.

[16] H. BrezIs, "Opérateurs Maximaux Monotones et Semi-groupes de Contractions dans les Espaces de Hilbert", North-Holland, 1973.

[17] H. BREZIS, "Analyse Fonctionnelle. Théorie et Applications", Collection Mathématiques Appliquées pour la Maîtrise, Masson, Paris, 1983.

[18] H. BRezis, M. MARCus and A. C. PonCE, Nonlinear elliptic equations with measures revisited, In: "Mathematical Aspects of Nonlinear Dispersive Equations", Ann. of Math. Stud., Vol. 163, Princeton Univ. Press, Princeton, NJ, 2007, 55-109.

[19] H. BREZIS and A. C. PONCE, Reduced measures for obstacle problems, Adv. Differential Equations 10 (2005), 1201-1234.

[20] H. BRezIS and W. Strauss, Semi-linear second-order elliptic equations in $L^{1}$, J. Math. Soc. Japan 25 (1973), 565-590.

[21] J. CRANK, "Free and Moving Boundary Problems", North-Holland, Amsterdam, 1977.

[22] G. Dal Maso, F. Murat, L. Orsina and A. Prignet, Renormalized solutions of elliptic equations with general measure data, Ann. Scuola Norm. Sup. Pisa Cl. Sci. (4) 28 (1999), 741-808.

[23] E. DiBenedetto and A. Friedman, The ill-posed Hele-Shaw model and the Stefan problem for supercooler water, Trans. Amer. Math. Soc. 282 (1984), 183-204.

[24] L. Dupaigne, A. C. Ponce and A. Porretta, Elliptic equations with vertical asymptotes in the nonlinear term, J. Anal. Math. 98 (2006), 349-396.

[25] G. DuvauX and J. L. Lions, "Inequalities in Mechanics and Physiscs", Springer-Verlag, 1976.

[26] C. M. Elliot and V. JAnOSKY, A variational inequality approach to the Hele-Shaw flow with a moving boundary, Proc. Roy. Soc. Edinburg Sect. A 88 (1981), 93-107.

[27] A. GMIRA and L. VÉRON, Boundary singularities of solutions of some nonlinear elliptic equations. Duke Math. J. 64 (1991), 271-324.

[28] J. Heinonen, T. Kilpeläinen and O. Martio, "Nonlinear Potential Theory of Degenerate Elliptic Equations", Oxford Mathematical Monographs, Oxford University Press, New York, 1993. 
[29] J. L. Lions, “Quelques Méthodes de Résolution de Problémes aux Limites non Linéaires”, Dunod-Gauthier-Vilars, Paris, 1968.

[30] M. MARCUS and L. VÉRON, Removable singularities and boundary traces, J. Math. Pures Appl. 80 (2001), 879-900.

[31] M. MARCUS and L. VÉRON, The precise boundary trace of solutions of a class of supercritical nonlinear equations, C. R. Math. Acad. Sci. Paris 344 (2007), 181-186.

[32] G. STAMPACCHIA, Le probléme de Dirichlet pour les équations elliptiques du second order à coefficients discontinus, Ann. Inst. Fourier (Grenoble) 15 (1965), 189-258.

[33] J. L. VÁzQUEZ, On a semilinear equation in $\mathbb{R}^{2}$ involving bounded measures, Proc. Roy. Soc. Edinburgh Sect. A 95 (1983), 181-202.

[34] W.P. ZIEMER, "Weakly Differentiable Functions", GTM 120, Springer-Verlag, 1989.

Departamento de Análisis Matemático Universitat de València

Dr. Moliner 50

46100 Burjassot (Valencia), Spain

Fuensanta.Andreu@uv.es

mazon@uv.es

toledojj@uv.es

LAMFA, CNRS-UMR 6140

Université de Picardie Jules Verne

33 rue Saint Leu

80038 Amiens, France

noureddine.igbida@u-picardie.fr 